Pacific

Journal of

Mathematics

\title{
AUTOMORPHISMS AND QUOTIENTS OF QUATERNIONIC FAKE QUADRICS
}

AMIR DŽAmbić AND XAVIER ROULlEAU 


\title{
AUTOMORPHISMS AND QUOTIENTS OF QUATERNIONIC FAKE QUADRICS
}

\author{
AMIR DŽAmbić AND XAVIER RoulleAu
}

\begin{abstract}
A $\mathbb{Q}$-homology quadric is a normal projective algebraic surface with the same Betti numbers as the smooth quadric in $\mathbb{P}^{3}$. A smooth $\mathbb{Q}$-homology quadric is either rational or of general type with vanishing geometric genus. Smooth minimal $\mathbb{Q}$-homology quadrics of general type are called fake quadrics. Here we study quaternionic fake quadrics, that is, fake quadrics whose fundamental group is an irreducible lattice in $\operatorname{PSL}_{2}(\mathbb{R}) \times \operatorname{PSL}_{2}(\mathbb{R})$ derived from a division quaternion algebra over a real number field. We provide examples of quaternionic fake quadrics $X$ with a nontrivial automorphism group $G$ and compute the invariants of the quotient $X / G$ and of its minimal desingularization $Z$. In this way we provide examples of singular $\mathbb{Q}$ homology quadrics and minimal surfaces $Z$ of general type with $q=p_{g}=0$ and $K^{2}=4$ or 2 which contain the maximal number of disjoint (-2)-curves. Conversely, we also show that if a smooth minimal surface of general type has the same invariant as $Z$ and same number of (-2)-curves, then we can construct geometrically a surface of general type with $c_{1}^{2}=8, c_{2}=4$.
\end{abstract}

\section{Introduction}

In this paper we will be interested in $\mathbb{Q}$-homology quadric surfaces, which are normal projective algebraic surfaces with the same Betti numbers as the quadric surface in $\mathbb{P}^{3}$, that is, $b_{1}=0$ and $b_{2}=2$. A smooth $\mathbb{Q}$-homology quadric $S$ has the following numerical invariants: $p_{g}(S)=q(S)=0, e(S)=c_{2}(S)=4$, and $c_{1}^{2}(S)=8$. By the classification theory of algebraic surfaces, such $S$ is either a Hirzebruch surface $\Sigma_{n}$ (with $\Sigma_{0}=\mathbb{P}^{1} \times \mathbb{P}^{1}$ ) or $S$ is of general type. The latter $S$ is either minimal or has at most one exceptional curve. Blowing down this $(-1)$-curve we obtain a fake projective plane, that is, a smooth minimal surface of general type with the same Betti numbers as the projective plane $\mathbb{P}^{2}$, being an example of a $\mathbb{Q}$-homology projective plane. By the analogy with fake projective planes, we define a fake quadric to be a minimal smooth $\mathbb{Q}$-homology quadric of general type (see [Barth et al. 2004, p. 231; Hirzebruch 1987, p. 780; Iskovskikh and Shafarevich 1989, p. 195]).

MSC2010: primary 14G35, 14J10, 14J29; secondary 14J50, 11F06, $11 \mathrm{R} 52$.

Keywords: $\mathbb{Q}$-homology quadrics, surfaces with $q=p_{g}=0$, fake quadrics, surfaces of general type, automorphisms. 
All known fake quadrics have $\mathbb{U} \times \mathbb{U}$, the product of two copies of the complex upper half plane, as the universal covering. Hence, such a fake quadric $X$ is of the form $X=\Gamma \backslash \mathbb{W} \times \mathbb{U}$, where $\Gamma$ is a torsion-free and cocompact discrete subgroup in Aut $(\mathbb{W} \times \mathbb{U})$, the group of holomorphic automorphisms of $\mathbb{U} \times \mathbb{W}$. Essentially, we distinguish between two classes of such quotients according to the structure of $\Gamma$.

One class of fake quadrics consists of surfaces $\Gamma \backslash \mathbb{H} \times \mathbb{M}$ with the property that the group $\Gamma$ is reducible. By reducible we mean that there exists a subgroup of finite index $\Gamma^{\prime}=\Gamma_{1} \times \Gamma_{2}$ of $\Gamma$ such that the group $\Gamma_{i}$ acts on $\mathbb{H}$ and $C_{i}=\mathbb{H} / \Gamma_{i}$ is a smooth algebraic curve. This case is now well understood and the full classification of these fake quadrics, named also fake quadrics isogenous to a higher product, has been achieved by Bauer, Catanese and Grunewald in [Bauer et al. 2008]. In practice, this classification and construction is done geometrically by classifying triples $\left(C_{1}, C_{2}, G\right)$ of two smooth curves $C_{i}$ of general type and a group $G$, such that $G$ acts faithfully and freely on the surface $C_{1} \times C_{2}$ and the quotient $\left(C_{1} \times C_{2}\right) / G$ has the asked invariants.

In this paper we will focus on fake quadrics of the other class, which we call quaternionic fake quadrics. These fake quadrics are Shimura surfaces, that is, quotients of $\mathbb{W} \times \mathbb{W}$ by cocompact irreducible arithmetic lattices $\Gamma$ in $\operatorname{Aut}(\mathbb{W}) \times$ Aut $(\mathbb{H})$, defined by an indefinite quaternion algebra over a totally real number field. Within the general framework of Prasad and Yeung on fake compact symmetric Hermitian spaces the quaternionic fake quadrics belong to the class of so-called arithmetic fake $A_{1}$; see [Prasad and Yeung 2012].

Using the previous work of Kuga, the first quaternionic fake quadrics have been constructed in [Shavel 1978]. We know that these surfaces are rigid and thus that there are only a finite number of them, but at the moment we do not have a complete list of all these surfaces. We have a list of commensurability classes of fake quadrics defined by quaternion algebras over quadratic fields (see [Džambić 2013]).

The situation for quaternionic fake quadrics is very similar to the case of fake projective planes. By the theorem of Klingler (and also Yeung), all fake projective planes are quotients of the 2-dimensional complex unit ball $\mathbb{B}^{2}$ by cocompact arithmetic lattices $\Gamma \subset \mathrm{PU}(2,1)$. This provides an arithmetic construction of these surfaces, but it is generally not easy to handle and construct these surfaces geometrically, for instance, as a quotient or ramified cover of some known surfaces.

In order to remedy this situation, Keum [2012; 2008; 2006] studied quotients of fake projective planes by groups of automorphisms. In this way, he obtained surfaces of general type with geometric genus $p_{g}=0$ and was able to rebuild a fake projective plane by only knowing the properties of the quotient surface.

The aim of this paper is to study automorphisms of quaternionic fake quadrics and the quotients of these surfaces by groups of automorphisms. Let $X=\Gamma \backslash \mathbb{H} \times \mathbb{\sharp}$ 
be a Shimura surface. We say that a curve $C \hookrightarrow X$ is a Shimura curve if it is a totally geodesic submanifold of $X$.

The first main result we obtain is the following:

Theorem A. An automorphism of a smooth Shimura surface $X=\Gamma \backslash \mathbb{W} \times \mathbb{W}$ has only finitely many fixed points or it is an involution whose fixed point set is a disjoint union of smooth Shimura curves.

An automorphism of a quaternionic fake quadric $X$ has only finitely many fixed points. There exist quaternionic fake quadrics $X$ with automorphism group isomorphic to

$$
\mathbb{Z} / 2 \mathbb{Z},(\mathbb{Z} / 2 \mathbb{Z})^{2}, \mathbb{D}_{4}, \mathbb{D}_{6}, \mathbb{D}_{8}, \text { or } \mathbb{D}_{10},
$$

where $\mathbb{D}_{n}$ is the dihedral group with order $2 n$.

Let us remark that the knowledge of surfaces of general type with $p_{g}=0$ and a large automorphism group can be interesting to check whether the Bloch conjecture holds (see, for example, [Inose and Mizukami 1979]). The computations in [Džambić 2013] lead us to the conjecture that the order of the automorphism group of a quaternionic fake quadric is always less or equal 24 (see Section 4).

The second aim of this paper is to study the minimal desingularization of the quotient of a quaternionic fake quadric by a group of automorphisms, in order to obtain new surfaces with $p_{g}=0$.

Theorem B. Let $X$ be a quaternionic fake quadric and $G$ a finite group of automorphisms of $X$. The minimal desingularization $Z$ of the quotient $X / G$ has the following numerical invariants:

\begin{tabular}{|c|c|c|c|c|c|}
\hline$G$ & $c_{1}^{2}(Z)$ & $c_{2}(Z)$ & Singularities on $X / G$ & Minimal & $\kappa(Z)$ \\
\hline $\mathbb{Z} / 2 \mathbb{Z}$ & 4 & 8 & $4 A_{1}$ & yes & 2 \\
$\mathbb{Z} / 3 \mathbb{Z}$ & 2 & 10 & $2 A_{3,1}+2 A_{2}$ & $?$ & 2 \\
$\mathbb{Z} / 6 \mathbb{Z}$ & -4 & 16 & $2 A_{6,1}+2 A_{5}$ & no & $?$ \\
$\mathbb{Z} / 8 \mathbb{Z}$ & -2 & 14 & $A_{8,3}+A_{8,5}$ & no & $?$ \\
$\mathbb{Z} / 10 \mathbb{Z}$ & -12 & 24 & $2 A_{10,1}+2 A_{9}$ & no & $?$ \\
$(\mathbb{Z} / 2 \mathbb{Z})^{2}$ & 2 & 10 & $6 A_{1}$ & yes & 2 \\
$\mathbb{D}_{4}$ & 0 & 12 & $4 A_{1}+A_{4,3}+A_{4,1}$ & no & $\geq 1$ \\
$\mathbb{D}_{8}$ & -1 & 13 & $4 A_{1}+A_{8,3}+A_{8,5}$ & no & $?$ \\
\hline
\end{tabular}

Here, $\kappa$ indicates the Kodaira dimension of the surface $Z$.

We obtain also results and restrictions for the groups $\mathbb{Z} / 4 \mathbb{Z}, \mathbb{Z} / 5 \mathbb{Z}$ and $\mathbb{D}_{3}$. We note that the surfaces of general type we obtain have vanishing geometric genus and could be therefore interesting from the point of view of the classification of surfaces with $p_{g}=0$. We intend to study these surfaces more closely, regarding, for instance, the fundamental groups in a future paper. 
A curve $C$ on a surface is called nodal if $C \simeq \mathbb{P}^{1}$ and $C^{2}=-2$. A nodal curve is the resolution of a nodal singularity. The surfaces $Z$ we obtain as the quotient of a fake quadric by an automorphism group $(\mathbb{Z} / 2 \mathbb{Z})^{n}, n \in\{1,2\}$ have the maximum number of nodal curves (the so-called Miyaoka bound [1984]). If minimal, the surfaces obtained by taking a quotient by the groups $\mathbb{Z} / 3 \mathbb{Z}$ and $\mathbb{D}_{3}$ have also the maximum number of quotient singularities. Similarly to Keum's construction of fake projective planes, we can reverse the construction.

Proposition C. Let $Z$ be a smooth minimal surface of general type with $q=p_{g}=0$.

(a) Suppose that $c_{1}^{2}=4,2$, or $1, \operatorname{Pic}(Z)$ has no 2-torsion, and that there is a birational map $Z \rightarrow Y$ onto a surface containing $8-c_{1}^{2}$ nodal singularities $A_{1}$. There exists a smooth minimal surface of general type $S$ with invariants $c_{1}^{2}=2 c_{2}=8$ and $a(\mathbb{Z} / 2 \mathbb{Z})^{m}$-cover $S \rightarrow Y$ ramified over the nodes, with $m$ such that $2^{m}=8 / c_{1}^{2}$.

(b) Suppose that $c_{1}^{2}=2, \operatorname{Pic}(Z)$ has no 3-torsion, and that there is a birational map $Z \rightarrow Y$ onto a surface with $2 A_{3,1}+2 A_{2}$ singularities. There exist a smooth surface $S$ with invariants $c_{1}^{2}=2 c_{2}=8$ and $a(\mathbb{Z} / 3 \mathbb{Z})$-cover $Z \rightarrow Y$ ramified over the singularities of $Y$.

The proof of part (a) of this proposition uses mainly the results of Dolgachev, Mendes Lopes, and Pardini [Dolgachev et al. 2002] and illustrates their theory. The proof of part (b) is more original because it mixes two types of singularities.

The paper is structured as follows: We begin recalling the known facts on quotients of surfaces (Section 2) and on quaternionic fake quadrics (Section 3). In Section 4, we provide examples of fake quadrics having a large group of automorphisms, we then compute the quotients surfaces (Section 5) and reverse the construction in the opposite direction: starting with a surface with the same invariants as the quotient, we construct a surface with $c_{1}^{2}=2 c_{2}=8$ (Section 6).

\section{Generalities on quotients of a surface}

In this section we recall results from the theory of quotient surface singularities and their resolution. The main reference for these topics is [Barth et al. 2004]; see also [Roulleau 2012].

Let $S$ be a smooth algebraic surface and let $G$ be a group of automorphisms acting on $S$. We denote by $S / G$ the quotient surface and by $\pi: Z \rightarrow S / G$ the minimal desingularization map. If $G=\langle\sigma\rangle$ is cyclic, we will often write $S / \sigma$ to denote the quotient $S /\langle\sigma\rangle$.

Proposition 2.4 (topological Lefschetz formula). Let $\sigma$ be an automorphism acting on $S$ and $S^{\sigma}$ the fixed point set of $\sigma$. We have 


$$
e\left(S^{\sigma}\right)=\sum_{j=0}^{j=4}(-1)^{j} \operatorname{Tr}\left(\sigma \mid H^{i}(S, \mathbb{Z})_{m t}\right),
$$

where $H^{i}(S, \mathbb{Z})_{m t}$ is the group $H^{i}(S, \mathbb{Z})$ modulo torsion.

Note that for a fake quadric $X$ we have $q=p_{g}=0$; thus

$$
H^{1}(X, \mathbb{Z})_{m t}=\{0\}, H^{2}(X, \mathbb{Z}) \otimes \mathbb{C}=H^{1}\left(X, \Omega_{X}\right) .
$$

Corollary 2.5. Let $X$ be a fake quadric and $\sigma$ an automorphism of order $n>1$ acting on $G$. We have $e\left(X^{\sigma}\right)=2$ or 4 . If $\sigma=\tau^{2}$ for an automorphism $\tau$ (for example, if $n$ is prime to 2$)$, we have $e\left(X^{\sigma}\right)=4$.

Proof. Since $X$ is a fake quadric, the space $H^{1}\left(X, \Omega_{X}\right)$ is 2-dimensional and is generated by the classes of 2-curves in the Néron-Severi group. As an automorphism preserves the canonical divisor, the invariant subspace of $H^{1}\left(X, \Omega_{X}\right)$ is at least one-dimensional. Therefore the trace of $\sigma$ on $H^{1}\left(X, \Omega_{X}\right)$ is 2 or 0 . If we suppose that this action is not trivial, then 2 divides the order of $\sigma$, moreover we see that the action of $\sigma^{2}$ is always trivial.

Let $\xi$ be a primitive $n$-th root of unity. Let us recall that for $1 \leq q \leq n-1$ coprime to $n$, the quotient of $\mathbb{C}^{2}$ by the action of

$$
(x, y) \rightarrow\left(\xi x, \xi^{q} y\right)
$$

has a unique singularity, called an $A_{n, q}$ singularity. For $n, m>0$ two numbers, we write $[n, m]$ for $n-1 / m$. The $A_{n, q}$ singularity is resolved by a Hirzebruch-Jung string (see [Barth et al. 2004]), that is, a chain of smooth rational curves $C_{1}, \ldots, C_{k}$ such that $C_{i}$ intersects $C_{i \pm 1}$ transversally in one point for $2 \leq i \leq k-1$ and $C_{i}^{2}=-n_{i}$ with integers $n_{i} \geq 2$ determined by the relation

$$
\frac{n}{q}=\left[n_{1},\left[n_{2}, \ldots,\left[n_{k-1}, n_{k}\right] \ldots\right]\right] .
$$

As is conventional, we denote $A_{n, n-1}$ by $A_{n-1}$.

Let $S$ be a surface with $p_{g}=q=0$ and let $\sigma$ be an automorphism of order $n \geq 2$ such that the fixed points of the $\sigma^{k}, k=1, \ldots, n-1$ are isolated.

Proposition 2.6 (holomorphic Lefschetz fixed point formula [Atiyah and Singer 1968, p. 567]). Let $S^{\sigma}$ be the fixed point set of $\sigma$. Then

$$
1=\sum_{s \in S^{\sigma}} \frac{1}{\operatorname{det}\left(1-d \sigma \mid T_{S, s}\right)},
$$

where $d \sigma_{s} \mid T_{S, s}$ denotes the action of $\sigma$ on the tangent space $T_{S, s}$. 
Suppose moreover that the automorphism $\sigma$ has prime order $p$. Let $\xi$ be a primitive $p$-th root of unity. Let $r_{i}$ be the number of isolated fixed points of $\sigma$ whose image in $S / \sigma$ are $A_{p, i}$ singularities.

Proposition 2.7 (Zhang's formula [2001, Lemma 1.6]). We have

$$
\sum_{i=1}^{p-1} r_{i} a_{i}(p)=1
$$

where

$$
a_{i}(p)=\frac{1}{p-1} \sum_{j=1}^{p-1} \frac{1}{\left(1-\xi^{j}\right)\left(1-\xi^{i j}\right)} .
$$

In particular, we have

$$
a_{1}(p)=\frac{1}{12}(5-p), \quad a_{2}(p)=\frac{1}{24}(11-p), \quad a_{3}(5)=\frac{1}{4}, \quad a_{4}(5)=\frac{1}{2} .
$$

Let $1 \leq i<p$ and $1 \leq k<p$ be such that $i k \equiv 1 \bmod p$. As $A_{p, i}=A_{p, k}$, the notations for $r_{i}$ and $r_{k}$ in Zhang's formula can be confusing. However, as $a_{i}(p)=a_{k}(p)$, there should be no trouble in taking the convention that $r_{i}+r_{k}$ is the total number of $A_{p, i}=A_{p, k}$ singularities, rather that choosing a representative $i$ or $k$ for every such pair $(i, k)$.

Let us recall that an automorphism of a vector space is called a reflection if all its eigenvalues but one are equal to 1 . Let $S$ be a surface and $G$ an automorphism group acting on $S$. Suppose that for every automorphism of $G$ the fixed point set is finite. Let $s$ be a fixed point of $G$; recall (see [Barth et al. 2004]):

Lemma 2.8. The action of the group $G$ on the tangent space $T_{S, s}$ is faithful and contains no reflections.

In particular, if $G$ is cyclic of order $n$, the singularity type of the image of the fixed point $s$ in the quotient $S / G$ is always $A_{n, q}$ with $q$ prime to $n$.

Lemma 2.9. The Euler number of $S / G$ is given by the formula

$$
e(S / G)=\frac{1}{|G|}\left(e(S)+\sum_{n \geq 2}(n-1) e\left(S_{n}\right)\right),
$$

where $S_{n}=\{s \in S|| \operatorname{Stab}(G, s) \mid=n\}$. The Euler number of the minimal resolution $Z$ is the sum of $e(S / G)$ and the number of irreducible components of the exceptional curves of the resolution $\pi: Z \rightarrow S / G$.

Let $C_{1}, \ldots, C_{k}$ be the irreducible components of the one dimensional fibers of $\pi: Z \rightarrow S / G$. We have the relations $K_{Z}=\pi^{*} K_{S / G}-\sum_{i=1}^{i=k} a_{i} C_{i}$, for rational numbers $a_{i}$ such that $K_{Z} C_{k}=-2-C_{k}^{2}$ and $C_{k} \pi^{*} K_{S / G}=0$. Moreover, we have the 
equality $K_{S / G}^{2}=K_{S}^{2} /|G|$, where $|G|$ is the order of $G$. As $K_{S}$ is ample, the canonical $\mathbb{Q}$-divisor $K_{S / G}$ is ample and $\pi^{*} K_{S / G}$ is nef. We remark also that $K_{Z}^{2} \leq K_{S / G}^{2}$.

Recall that the Kähler lemma implies that for a dominant rational map between varieties, the pull back map among the spaces of sections of sheaves of holomorphic forms are injective, therefore we obtain (see also [Roulleau 2012] for a proof in the case of surfaces):

Lemma 2.10. Let $S$ be a surface with $q=p_{g}=0$. The minimal resolution $Z$ of the quotient of $S$ by a group $G$ has also $q=p_{g}=0$.

Suppose that $S$ is moreover minimal of general type and the fixed points of automorphisms in $G$ are isolated.

Lemma 2.11. If $K_{Z}^{2}=0$, the surface $Z$ has Kodaira dimension $\kappa \geq 1$. If $K_{Z}^{2}>0$, the surface $Z$ has Kodaira dimension $\kappa=2$.

Proof. (We follow the ideas from [Keum 2008].) The quotient surface has $q=p_{g}=0$ and thus $\chi\left(\mathcal{O}_{Z}\right)=1$. Let $m \geq 1$ be an integer. Then

$$
-m K_{Z} \pi^{*} K_{S / G}=-m K_{S / G}^{2}=-\frac{8}{|G|} m<0 ;
$$

therefore $H^{0}\left(Z,-m K_{Z}\right)=\{0\}$ for every $m \geq 1$. Let $m \geq 2$; then from the Serre duality and Riemann-Roch we obtain

$$
H^{0}\left(Z, m K_{Z}\right)=\chi\left(\mathcal{O}_{Z}\right)+\frac{m(m-1)}{2} K_{Z}^{2}+h^{1}\left(Z, m K_{Z}\right) .
$$

If $K_{Z}^{2}>0$, it immediately follows that $Z$ is of general type. If $K_{Z}^{2}=0$, the surface has $h^{0}\left(Z, 2 K_{Z}\right) \neq 0$ and cannot be rational by the Castelnuovo criterion. Moreover, as $\chi=1$ it cannot be a ruled surface. Suppose that $Z$ is an Enriques surface. As $K_{Z}^{2}=0$, it is a minimal surface, but this is impossible because $h^{0}\left(Z, 3 K_{Z}\right) \neq 0$; therefore $\kappa>0$.

\section{Automorphisms of smooth Shimura surfaces and generalities on quaternionic fake quadrics}

Let us give a more detailed description of Shimura surfaces and quaternionic fake quadrics. First, recall that a lattice $\Gamma<\mathrm{PSL}_{2}(\mathbb{R}) \times \mathrm{PSL}_{2}(\mathbb{R}) \cong$ Aut $\mathbb{W} \times$ Aut $\mathbb{M}$ is irreducible if it is not commensurable with a product $\Gamma_{1} \times \Gamma_{2}$ of two discrete subgroups $\Gamma_{1}, \Gamma_{2} \subset \operatorname{PSL}_{2}(\mathbb{R})$. Equivalently, the image of $\Gamma$ under the projection onto one of the factors $\mathrm{PSL}_{2}(\mathbb{R})$ is a dense subgroup of $\mathrm{PSL}_{2}(\mathbb{R})$. Irreducible lattices in $\mathrm{PSL}_{2}(\mathbb{R}) \times \mathrm{PSL}_{2}(\mathbb{R})$ can be constructed arithmetically in the following way. Let $k$ be a totally real number field of degree $g=[k: \mathbb{Q}] \geq 2$, and let 
$B=(\alpha, \beta)_{k}:=\langle 1, i, j, i j\rangle_{k}$, with $i^{2}=\alpha \in k, j^{2}=\beta \in k, i j=-j i$, be a quaternion algebra over $k$ such that

$$
B \otimes_{\mathbb{Q}} \mathbb{R}=\prod_{\rho \in \operatorname{Hom}(k, \mathbb{R})} B^{\rho} \cong M_{2}(\mathbb{R}) \times M_{2}(\mathbb{R}) \times \underbrace{H_{\mathbb{R}} \times \cdots \times H_{\mathbb{R}}}_{g-2} .
$$

Here, $B^{\rho}=\left(\alpha^{\rho}, \beta^{\rho}\right)_{\mathbb{R}}$ and $H_{\mathbb{R}}=(-1,-1)_{\mathbb{R}}$ denotes the skew field of Hamiltonian quaternions. Let $\mathcal{O}_{k}$ be the ring of integers of $k$ and $\mathcal{O}$ a maximal order in $B$, that is, a maximal subring of $B$ which is a complete $\mathcal{O}_{k}$-lattice in $B$. Finally, let $\mathcal{O}^{1}$ be the subgroup of all elements in $\mathcal{O}$ of reduced norm one.

The isomorphism (3-1) induces an embedding of $\mathcal{O}^{1}$ into $\mathrm{SL}_{2}(\mathbb{R}) \times \mathrm{SL}_{2}(\mathbb{R})$ by taking the element $\gamma \in \mathcal{O}^{1}$ to the pair $\left(\gamma^{\rho_{1}}, \gamma^{\rho_{2}}\right) \in \mathrm{SL}_{2}(\mathbb{R}) \times \mathrm{SL}_{2}(\mathbb{R})$, where $\gamma^{\rho_{i}}$ is the image of $\gamma$ in $B^{\rho_{i}}$. The group $\mathcal{O}^{1}$ then acts on $\mathbb{H} \times \mathbb{H}$ as a group of fractional linear transformations. Namely, if $(z, w) \in \mathbb{W} \times \mathbb{W}$ is a point and an element $\gamma \in \mathcal{O}^{1}$ is identified with two matrices $\gamma^{\rho_{1}}$ and $\gamma^{\rho_{2}} \in \mathrm{SL}_{2}(\mathbb{R})$, then

$$
\gamma(z, w)=\left(\gamma^{\rho_{1}} z, \gamma^{\rho_{2}} w\right) .
$$

After dividing out by the ineffective kernel, one considers the group

$$
\Gamma_{\mathcal{O}}^{1}=\mathcal{O}^{1} /\{ \pm 1\} \subset \operatorname{PSL}_{2}(\mathbb{R}) \times \operatorname{PSL}_{2}(\mathbb{R})
$$

and it can be proven that $\Gamma_{\mathcal{O}}^{1}$ is an irreducible lattice in $\mathrm{PSL}_{2}(\mathbb{R}) \times \mathrm{PSL}_{2}(\mathbb{R})$. Moreover, this lattice is cocompact if and only if $B$ is a division quaternion algebra (see [Vignéras 1980, p. 104]). In general we say that a subgroup $\Gamma \subset \mathrm{PSL}_{2}(\mathbb{R}) \times \mathrm{PSL}_{2}(\mathbb{R})$ is an arithmetic lattice if there exists $k, B, \rho_{1}, \rho_{2}, \mathcal{O}$ as above such that $\Gamma$ is commensurable with $\Gamma_{\mathcal{O}}^{1}$. Since $\mathrm{PSL}_{2}(\mathbb{R}) \times \mathrm{PSL}_{2}(\mathbb{R})$ is a semisimple real Lie group of real rank 2, the famous arithmeticity theorem of Margulis [1991, Theorem (A), p. 298] (or see [Zimmer 1984, Theorem 6.1.2, p. 114]) states that any irreducible lattice $\Gamma$ in $\mathrm{PSL}_{2}(\mathbb{R}) \times \mathrm{PSL}_{2}(\mathbb{R})$ is an arithmetic lattice.

Let $\Gamma$ be irreducible and cocompact (arithmetic) lattice in $\mathrm{PSL}_{2}(\mathbb{R}) \times \mathrm{PSL}_{2}(\mathbb{R})$ and $X_{\Gamma}:=\Gamma \backslash \mathbb{H} \times \mathbb{W}$ be the orbit space of the discontinuous action of $\Gamma$ on $\mathbb{H} \times \mathbb{H}$. Then, there is a natural structure of compact algebraic surface on $X_{\Gamma}$. Such a surface $X_{\Gamma}$ is called (compact) Shimura surface and can be seen as the compact analog of a Hilbert modular surface. We know that $X_{\Gamma}$ is smooth if $\Gamma$ is torsion free. The numerical invariants of a smooth $X_{\Gamma}$ are computed in [Matsushima and Shimura 1963]; see also [Shavel 1978]. It follows that a smooth $X_{\Gamma}$ is a fake quadric if and only if $c_{2}\left(X_{\Gamma}\right)=4$ (see [Shavel 1978]).

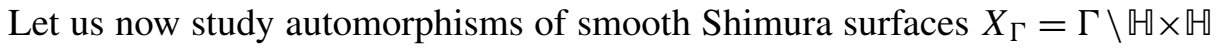

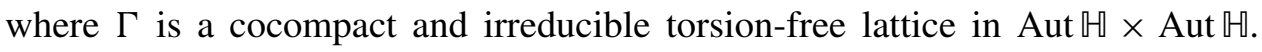
Let $\mu: \mathbb{W} \times \mathbb{W} \rightarrow \mathbb{W} \times \mathbb{W}$ be the involution exchanging the two factors. The group $\operatorname{Aut}(\mathbb{W} \times \mathbb{U})$ is the semidirect product of Aut $\mathbb{W} \times$ Aut $\mathbb{W}$ by the group generated by $\mu$. Let $N \Gamma$ (resp. $\left.N_{0} \Gamma\right)$ be the normalizer of $\Gamma$ in $\operatorname{Aut}(\mathbb{H} \times \mathbb{H})($ resp. in Aut $\mathbb{W} \times$ Aut $\mathbb{U})$; 
$N_{0} \Gamma$ is a subgroup of $N \Gamma$ of index 1 or 2 . The following result is crucial for our computations.

Theorem 3.12. The automorphism group of the smooth Shimura surface $X_{\Gamma}$ is (isomorphic to) $\mathrm{N} \Gamma / \Gamma$. An automorphism has only finitely many fixed points or it is an involution whose fixed point set is a union of smooth Shimura curves.

There exists an involution with a purely one-dimensional fixed point set if and only if $N \Gamma \neq N_{0} \Gamma$.

An automorphism of a fake quadric has only finitely many fixed points.

Proof. Since $\mathbb{U} \times \mathbb{W}$ is the universal covering of $X_{\Gamma}$, every automorphism $\sigma$ of $X_{\Gamma}$ lifts to an automorphism $\gamma$ of $\mathbb{H} \times \mathbb{H}$; this $\gamma$ normalizes $\Gamma$ and two elements $\gamma, \gamma^{\prime}$ both represent $\sigma$ if and only if $\gamma^{-1} \gamma^{\prime} \in \Gamma$. Hence, $\sigma$ defines an element $\gamma \Gamma$ of the group $N \Gamma / \Gamma$. Conversely, for a class $\gamma \Gamma \in N \Gamma / \Gamma$, the map $\sigma: X \rightarrow X$ defined by $\sigma(\Gamma x)=\gamma \Gamma x=\gamma \Gamma \gamma^{-1} \gamma x=\Gamma \gamma x$ is an automorphism of $X$. We thus proved that $\operatorname{Aut}(X)=N \Gamma / \Gamma$.

We say that $\sigma \in \operatorname{Aut}(X)=N \Gamma / \Gamma$, with $\sigma=\gamma \Gamma \in N \Gamma / \Gamma$, is a factor preserving automorphism if $\gamma$ is in $N_{0} \Gamma$.

Let us denote by $\mathbb{F}_{\Gamma}$ a fundamental domain in $\mathbb{H} \times \mathbb{U}$ of $\Gamma$. Let $\sigma \in \operatorname{Aut}(X)$ be a nontrivial factor preserving automorphism and let $s$ be a fixed point, with representative $\left(z_{1}, z_{2}\right)$ in $\mathbb{F}_{\Gamma}$. Let $\gamma \in N_{0} \Gamma$ be a representative of $\sigma$ such that $\gamma\left(z_{1}, z_{2}\right)=\left(\gamma^{\rho_{1}} z_{1}, \gamma^{\rho_{2}} z_{2}\right)=\left(z_{1}, z_{2}\right)$. The point $s$ is an isolated fixed point of $\sigma$ if and only if $\gamma$ has finitely many fixed points in $\mathbb{F}_{\Gamma}$.

Since $\left(\gamma^{\rho_{1}} z_{1}, \gamma^{\rho_{2}} z_{2}\right)=\left(z_{1}, z_{2}\right), z_{1}$ is a fixed point of $\gamma^{\rho_{1}}$ and $z_{2}$ is a fixed point of $\gamma^{\rho_{2}}$. The only automorphisms of $\mathbb{W}$ with fixed points in $\mathbb{U}$ are elliptic transformations or the identity. An elliptic transformation has a unique fixed point in $\mathbb{H}$.

By Shimizu's theorem [1963, Theorem 2], $\gamma^{\rho_{1}}$ is trivial if and only if $\gamma^{\rho_{2}}$ is trivial. Since we supposed that $\sigma$ is nontrivial, at least one - and thus both - of the $\gamma^{\rho_{i}}$ are elliptic elements of $\operatorname{PSL}_{2}(\mathbb{R})$. Thus the point $\left(z_{1}, z_{2}\right)$ is the unique fixed point of $\gamma$ in $\mathbb{H} \times \mathbb{U}$, therefore the point $s$ is an isolated fixed point of $\sigma$.

Suppose now that $\sigma \in \operatorname{Aut}(X)$ is not a factor preserving automorphism. Let $\gamma^{\prime} \in N \Gamma$ a representative of $\sigma \in N \Gamma / \Gamma$. There exists $\gamma=\left(\gamma_{1}, \gamma_{2}\right) \in$ Aut $\mathbb{H} \times$ Aut $\mathbb{U}$ such that $\gamma^{\prime}=\gamma \mu \in \operatorname{Aut}(\mathbb{H} \times \mathbb{H})$. Suppose that $\sigma$ has an infinite number of fixed points. Then by the above discussion, the factor preserving automorphism $\sigma^{2}$ (with representative $\left.(\gamma \mu)^{2}=\left(\gamma_{1} \gamma_{2}, \gamma_{2} \gamma_{1}\right)\right)$ must be the identity and $\left(\gamma_{1} \gamma_{2}, \gamma_{2} \gamma_{1}\right)$ must be in $\Gamma$. Let $s=\Gamma\left(z_{1}, z_{2}\right)$ be a fixed point of $\sigma$. There exists $\lambda \in \Gamma$ such that

$$
\left(\gamma_{1} z_{2}, \gamma_{2} z_{1}\right)=\lambda\left(z_{1}, z_{2}\right) \text {. }
$$

After the change of the representative $\gamma^{\prime}$ by $\lambda^{-1} \gamma^{\prime}$, we can assume that $\lambda=1$, thus $z_{2}=\gamma_{2} z_{1}, \gamma_{1} \gamma_{2} z_{1}=z_{1}$ and $\gamma_{1} \gamma_{2} z_{2}=z_{2}$. Since $\left(\gamma_{1} \gamma_{2}, \gamma_{2} \gamma_{1}\right)$ is in the group $\Gamma$ in 
which a nontrivial element has no fixed points, we obtain that $\gamma_{2} \gamma_{1}=\gamma_{1} \gamma_{2}=1$. Since $\gamma_{1} \gamma_{2}=1$, the point $\left(t, \gamma_{2} t\right)$ (for $t \in \mathbb{U}$ ) satisfies

$$
\gamma^{\prime}\left(t, \gamma_{2} t\right)=\left(t, \gamma_{2} t\right)
$$

therefore there are no isolated fixed points for $\sigma$ and its fixed point set is purely one-dimensional. The image of the disk $\Delta=\left\{\left(t, \gamma_{2} t\right) \mid t \in \mathbb{U}\right\}$ in $X$ is a smooth Shimura curve (see, for instance, [Granath 2002, Chapter 7]) fixed by $\sigma$.

Assume now that $X_{\Gamma}$ is a quaternionic fake quadric and that the fixed locus $C$ of $\sigma$ is a smooth curve. The topological Lefschetz formula (see Corollary 2.5) implies that the genus of the irreducible components of $C$ is negative, thus the automorphism has only a finite number of fixed points.

Remark 3.13. Note that according to Theorem 3.12 and the proof of Corollary 2.5, the quotient of a quaternionic fake quadric by a group $G$ is a $\mathbb{Q}$-homology quadric if and only if each automorphism $\sigma \in G$ has 4 fixed points, otherwise this quotient is a $\mathbb{Q}$-homology projective plane. All the cyclic groups $G$ which we will study in Section 5 give examples of $\mathbb{Q}$-homology quadrics, with the only possible exception for automorphisms of order 4 .

\section{Quaternionic fake quadrics with nontrivial automorphism groups}

As already mentioned, a series of examples of quaternionic fake quadrics has been constructed in [Shavel 1978]. There, the author concentrates on arithmetic lattices $\Gamma \supseteq \Gamma_{\mathcal{O}}^{1}$ which are defined by quaternion algebras over real quadratic fields of class number one. More recently, in [Džambić 2013], more examples of quaternionic quadrics associated with quaternion algebras over quadratic fields have been found. In this section we will give examples of some known quaternionic fake quadrics together with their automorphism groups. We refer the reader to [Vignéras 1980] and [Deuring 1968] for generalities on arithmetic theory of quaternion algebras.

Let us first make a few general observations, before we discuss the examples in detail. For technical reasons it is more practical to consider the group $\mathrm{PGL}_{2}^{+}(\mathbb{R}) \times \mathrm{PGL}_{2}^{+}(\mathbb{R})$, where $\mathrm{PGL}_{2}^{+}(\mathbb{R})=\mathrm{GL}_{2}^{+}(\mathbb{R}) / \mathbb{R}^{*}$ and $\mathrm{GL}_{2}^{+}(\mathbb{R})$ is the group of all $2 \times 2$ matrices with positive determinant, instead of $\mathrm{PSL}_{2}(\mathbb{R}) \times \mathrm{PSL}_{2}(\mathbb{R})$. We identify $\mathrm{PGL}_{2}^{+}(\mathbb{R}) \times \mathrm{PGL}_{2}^{+}(\mathbb{R})$ with the group Aut $\mathbb{H} \times$ Aut $\mathbb{H}$ of factor preserving holomorphic automorphisms.

From the point of view of Theorem 3.12 we will be interested only in automorphism subgroups $G \leq N \Gamma / \Gamma=\operatorname{Aut}\left(X_{\Gamma}\right)$ of factor preserving automorphisms, that

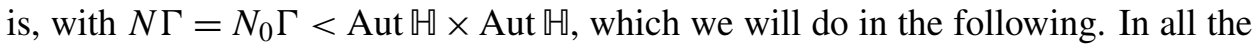
considered examples the normalizers $N \Gamma$ will be normalizers of maximal orders and all such lattices can be described arithmetically as follows (see [Borel 1981]). 
If $X_{\Gamma}$ is a quaternionic fake quadric, there is an associated tuple $\left(k, \rho_{1}, \rho_{2}, B, \mathcal{O}\right)$ as described in Section 3. The quaternion algebra $B$ is for fixed $\rho_{1}, \rho_{2}$ uniquely determined (up to isomorphism) by the reduced discriminant $d_{B}=v_{1} \cdots v_{r}$, the formal product over finite places $v_{i}$ of $k$ where $B$ is ramified, that is, $B \otimes_{k} k_{v_{i}} ¥ M_{2}\left(k_{v_{i}}\right)$. This is a special case of H. Hasse's deep classification theorem for simple algebras over number fields (see [Deuring 1968, VII.5, Satz 9, p. 119] or [Vignéras 1980, Chapitre III, Théorème 3.1, p. 74] ). Hence, $\left(k, \rho_{1}, \rho_{2}, B, \mathcal{O}\right)=\left(k, \rho_{1}, \rho_{2}, d_{B}, \mathcal{O}\right)$. In the following we will often abbreviate such a datum which determines the quaternion algebra $B$ with $B\left(k, d_{B}\right)$ or $B\left(k, v_{1} \cdots v_{r}\right)$. Let us fix a datum $B\left(k, v_{1} \cdots v_{r}\right)$ and let $B^{+}$be the group of all $x \in B^{*}$ such that the reduced norm $\operatorname{Nrd}(x)$ is totally positive. It is known that

$$
N \Gamma_{\mathcal{O}}^{+}=\left\{x \in B^{+} \mid x \mathcal{O} x^{-1}=\mathcal{O}\right\} / k^{*}
$$

is a maximal lattice. $N \Gamma_{\mathcal{O}}^{+}$contains $\Gamma_{\mathcal{O}}^{1}$ and $\Gamma_{\mathcal{O}}^{1}$ is normal in $N \Gamma_{\mathcal{O}}^{+}$with $N \Gamma_{\mathcal{O}}^{+} / \Gamma_{\mathcal{O}}^{1} \cong$ $(\mathbb{Z} / 2 \mathbb{Z})^{l}$ an elementary abelian 2-group with $l \geq r$ and $r$ is the number of ramified places in $B$ (see [Shavel 1978], for instance). If the class number of $k$ is one (as will be the case in all the considered examples) there is an alternative description of $N \Gamma_{\mathcal{O}}^{+}$as

$$
\begin{aligned}
N \Gamma_{\mathcal{O}}^{+}=\left\{\alpha=\varrho_{1}^{\epsilon_{1}} \cdots \varrho_{r}^{\epsilon_{r}} \lambda \tau \in B^{*} \mid\right. & \operatorname{Nrd}(\alpha) \text { totally positive, } \\
\tau & \left.\in k^{*}, \lambda \in \mathcal{O}^{*}, \epsilon_{i} \in\{0,1\}, \operatorname{Nrd}\left(\varrho_{i}\right) \text { divides } d_{B}\right\} / k^{*}
\end{aligned}
$$

(see [Shavel 1978, p. 223]). It follows that a quaternionic fake quadric $X_{\Gamma}$ with $\Gamma \supseteq \Gamma_{\mathcal{O}}^{1}$ will have an elementary abelian 2-group as the automorphism group $\operatorname{Aut}\left(X_{\Gamma}\right)$. All Shavel's examples will provide such automorphism groups.

A fake quadric with automorphism group $\mathbb{Z} / \mathbf{2} \mathbb{Z}$. There are examples of quaternionic fake quadrics $X_{\Gamma}$ whose automorphism group is $\mathbb{Z} / 2 \mathbb{Z}$ and, as mentioned above, they already appear in [Shavel 1978].

For example, let $k=\mathbb{Q}(\sqrt{2})$ and let $B=B\left(k, \mathfrak{p}_{3} \mathfrak{p}_{7}\right)$ be the (unique) quaternion algebra over $k$ which is ramified exactly at the two finite primes $\mathfrak{p}_{3}$ and $\mathfrak{p}_{7}$ of $k$ lying over the rational primes 3 and 7 respectively. Since $k$ has the class number one, there is the unique (up to conjugation) maximal order $\mathcal{O}$ in $B$. Consider the group $\Gamma_{\mathcal{O}}^{1}$. By [Shavel 1978, Proposition 4.7], $X_{\Gamma_{\mathcal{O}}^{1}}$ is smooth. By the already mentioned general result of Matsushima and Shimura [1963], $q\left(X_{\Gamma_{\mathcal{O}}^{1}}\right)=0$. The Chern number $c_{2}\left(X_{\Gamma_{\mathcal{O}}^{1}}\right)$ is computed via the volume formula of Shimizu (see [Shavel 1978, Theorem 3.1]). Since the prime 3 is inert and 7 is decomposed in $k$, this formula gives $c_{2}\left(X_{\Gamma_{\mathcal{O}}^{1}}\right)=8$. The normalizer of $\Gamma_{\mathcal{O}}^{1}$ is $N \Gamma_{\mathcal{O}}^{+}$and by [Shavel 1978, Proposition 1.3 and Proposition 1.4], we have

$$
\operatorname{Aut}\left(X_{\Gamma_{\mathcal{O}}^{1}}\right) \cong L_{1} / L_{2}=\left\langle\left[\mathfrak{p}_{3}\right],\left[\mathfrak{p}_{7}\right]\right\rangle \cong(\mathbb{Z} / 2 \mathbb{Z})^{2},
$$


where $L_{1}$ is the group of principal fractional ideals of type $\left(\mathfrak{p}_{3}\right)\left(\mathfrak{p}_{7}\right) I^{2}$ ( $I$ a principal fractional ideal) for which one can find a totally positive generator and $L_{2}$ consists of all principal ideals of type ( $\left.a^{2}\right)$ with $a \in k$ (see also [Shimura 1967, Section 3.12]). Let $\Gamma_{\mathfrak{p}_{3}}$ be the kernel of the canonical homomorphism

$$
N \Gamma_{\mathcal{O}}^{+} \longrightarrow L_{1} / L_{2} \longrightarrow\left\langle\left[\mathfrak{p}_{7}\right]\right\rangle .
$$

By Shavel's criterion [1978, Theorem 4.11], $\Gamma_{\mathfrak{p}_{3}}$ is torsion free and as $\left[\Gamma_{\mathfrak{p}_{3}}: \Gamma_{\mathcal{O}}^{1}\right]=2$, $X_{\Gamma_{\mathfrak{p}_{3}}}$ is a fake quadric with $\operatorname{Aut}\left(X_{\Gamma_{\mathfrak{p}_{3}}}\right) \cong \mathbb{Z} / 2 \mathbb{Z}$.

A fake quadric with automorphism group $(\mathbb{Z} / 2 \mathbb{Z})^{\mathbf{2}}$. Consider again $k=\mathbb{Q}(\sqrt{2})$ and now the quaternion algebra $B=B\left(k, \mathfrak{p}_{2} \mathfrak{p}_{5}\right)$ over $k$ which is ramified exactly at the two finite places $\mathfrak{p}_{2}$ and $\mathfrak{p}_{5}$. Again there is the unique maximal order $\mathcal{O}$ in $B$ and as in the previous example, Shavel's results show that $X_{\Gamma_{\mathcal{O}}^{1}}$ is smooth. The prime 2 is ramified and 5 is inert in $k$ and therefore Shimizu's volume formula gives $c_{2}\left(X_{\Gamma_{\mathcal{O}}^{1}}\right)=4$. Hence $X_{\Gamma_{\mathcal{O}}^{1}}$ is a fake quadric. With the same arguments as in the previous example $\operatorname{Aut}\left(X_{\Gamma_{\mathcal{O}}^{1}}\right)$ is isomorphic to $(\mathbb{Z} / 2 \mathbb{Z})^{2}$.

A fake quadric with automorphism group of order 20. Consider $k=\mathbb{Q}(\sqrt{5})$ and the quaternion algebra $B=B\left(k, \mathfrak{p}_{2} \mathfrak{p}_{5}\right)$ over $k$ which is ramified exactly at the primes $\mathfrak{p}_{2}$ and $\mathfrak{p}_{5}$. In this case the group $\Gamma_{\mathcal{O}}^{1}$ (where $\mathcal{O}$ is again a maximal order in $B$ ), contains torsion elements of order 5 and no other torsions (see [Shavel 1978, Proposition 4.7 and Theorem 4.8]). ${ }^{1}$ Volume formula of Shimizu gives in this case $c_{2}\left(X_{\Gamma_{\mathcal{O}}^{1}}\right)=4 / 5$. Let us now give a torsion-free subgroup $\Gamma<\Gamma_{\mathcal{O}}^{1}$ of index 5 . The corresponding surface $X_{\Gamma}$ will be a fake quadric. Since $\mathfrak{p}_{2}$ is ramified in $B$, there is a prime ideal $\mathfrak{P}_{2}$ in $\mathcal{O}$ lying over $\mathfrak{p}_{2}$ and satisfying $\mathfrak{P}_{2}^{2}=\mathfrak{p}_{2} \mathcal{O}$. Let

$$
\mathcal{O}^{1}\left(\mathfrak{P}_{2}\right)=\left\{x \in \mathcal{O}^{1} \mid x \equiv 1 \bmod \mathfrak{P}_{2}\right\}
$$

and $\Gamma_{\mathcal{O}}^{1}\left(\mathfrak{P}_{2}\right)$ the image of $\mathcal{O}^{1}\left(\mathfrak{P}_{2}\right)$ in $\Gamma_{\mathcal{O}}^{1}$. The group $\Gamma_{\mathcal{O}}^{1}\left(\mathfrak{P}_{2}\right)$ is a normal subgroup in $\Gamma_{\mathcal{O}}^{1}$ and the index can be computed via the localization of $B$ at $\mathfrak{p}_{2}$. Namely, observe first that $\Gamma_{\mathcal{O}}^{1} / \Gamma_{\mathcal{O}}^{1}\left(\mathfrak{P}_{2}\right)$ is isomorphic to the factor group $\mathcal{O}^{1} / \mathcal{O}^{1}\left(\mathfrak{P}_{2}\right)$. This is because -1 is in $\mathcal{O}^{1}\left(\mathfrak{P}_{2}\right)$. Let $\mathcal{O}_{\mathfrak{p}_{2}}$ be the maximal order in $B_{\mathfrak{p}_{2}}$, that is, $\mathcal{O}_{\mathfrak{p}_{2}}=\mathcal{O} \otimes_{\mathscr{O}_{k}} \mathcal{O}_{k_{\mathfrak{p}_{2}}}$, where $\mathcal{O}_{k_{\mathfrak{p}_{2}}}$ is the ring of integers in $k_{\mathfrak{p}_{2}}$. Its maximal ideal $\widehat{\mathfrak{P}}_{2}$ is the topological closure of $\mathfrak{P}_{2}$. By the strong approximation property, $\mathcal{O}^{1} / \mathcal{O}^{1}\left(\mathfrak{P}_{2}\right) \cong \mathcal{O}_{\mathfrak{p}_{2}}^{1} / \mathcal{O}_{\mathfrak{p}_{2}}^{1}\left(\widehat{\mathfrak{P}}_{2}\right)$. Note that $B_{\mathfrak{p}_{2}}^{1}=\mathcal{O}_{\mathfrak{p}_{2}}^{1}$, since $\mathcal{O}_{\mathfrak{p}_{2}}$ is the subring of $B_{\mathfrak{p}_{2}}$ consisting of elements whose reduced norm is less or equal 1. We use a theorem of C. Riehm [1970, Theorem 7] by which

$$
\mathcal{O}_{\mathfrak{p}_{2}}^{1} / \mathcal{O}_{\mathfrak{p}_{2}}^{1}\left(\widehat{\mathfrak{P}}_{2}\right) \cong \operatorname{ker}\left(\left(\mathcal{O}_{\mathfrak{p}_{2}} / \widehat{\mathfrak{P}}_{2}\right)^{*} \stackrel{\mathrm{Nr}}{\longrightarrow}\left(\mathcal{O}_{k_{\mathfrak{p}_{2}}} / \mathfrak{p}_{2}\right)^{*}\right) \cong \operatorname{ker}\left(\mathbb{F}_{16}^{*} \stackrel{\mathrm{Nr}}{\longrightarrow} \mathbb{F}_{4}^{*}\right) \cong \mathbb{Z} / 5 \mathbb{Z}
$$

\footnotetext{
${ }^{1}$ In Theorem 4.8 of [Shavel 1978], the symbol $\left(\frac{\bar{p}}{)}\right.$ for $p=2$ should be read as the Kronecker symbol; that is, $\left(\frac{d}{2}\right)=1 \Leftrightarrow d \equiv \pm 1 \bmod 8$ and $=-1 \Leftrightarrow d \equiv \pm 3 \bmod 8$.
} 
(Here $\mathrm{Nr}$ is the surjective homomorphism of multiplicative groups arising from the norm map for the field extension $\mathbb{F}_{16} / \mathbb{F}_{4}$.) Since $\Gamma_{\mathcal{O}}^{1}\left(\mathfrak{P}_{2}\right)$ is embedded in $\mathcal{O}_{\mathfrak{p}_{2}}^{1}\left(\widehat{\mathfrak{P}}_{2}\right) / \pm 1$ and the latter group is a pro-2-group (again by [Riehm 1970]) it cannot contain elements of order 5. Therefore, $\Gamma_{\mathcal{O}}^{1}\left(\mathfrak{P}_{2}\right)$ is a torsion-free group and $X_{\Gamma_{\mathcal{O}}^{1}}^{1}\left(\mathfrak{P}_{2}\right)$ is a fake quadric. Since $\Gamma_{\mathcal{O}}^{1}$ contains a 5 -torsion and $\Gamma_{\mathcal{O}}^{1}$ normalizes $\Gamma_{\mathcal{O}}^{1}\left(\mathfrak{P}_{2}\right), X_{\Gamma_{\mathcal{O}}^{1}\left(\mathfrak{P}_{2}\right)}$ contains an automorphism of order 5 . In order to determine the full automorphism group $\operatorname{Aut}\left(X_{\Gamma_{\mathcal{O}}^{1}\left(\mathfrak{P}_{2}\right)}\right)$ we first need to find the normalizer of $\Gamma_{\mathcal{O}}^{1}\left(\mathfrak{P}_{2}\right)$. By definition, elements of $N \Gamma_{\mathcal{O}}^{+}$normalize $\mathcal{O}$, that is, $x \mathcal{O} x^{-1}=\mathcal{O}$. Let $\gamma \in \Gamma_{\mathcal{O}}^{1}\left(\mathfrak{P}_{2}\right)$. Since the class number of $k$ is one, every two-sided $\mathcal{O}$-ideal is principal and we can choose $\Pi_{2} \in \mathcal{O}$ such that $\Pi_{2} \mathcal{O}=\mathfrak{P}_{2}$. Moreover, as $\mathfrak{P}_{2}$ is uniquely determined by the property that the $\mathrm{O}_{k}$-ideal $\operatorname{Nrd}\left(\mathfrak{P}_{2}\right)$ is $\mathfrak{p}_{2}$, we can choose $\Pi_{2}$ such that $\operatorname{Nrd}\left(\Pi_{2}\right)=2$. Then $\gamma= \pm\left(1+m \Pi_{2}\right)$ with $m \in \mathcal{O}$. For $x \in N \Gamma_{\mathcal{O}}^{+}$we have $x \gamma x^{-1}=1+x m \Pi_{2} x^{-1}=1+m^{\prime} x \Pi_{2} x^{-1}$ with some $m^{\prime} \in \mathcal{O}$. The element $x \Pi_{2} x^{-1}$ lies in $\mathcal{O}$ and $\operatorname{Nrd}\left(x \Pi_{2} x^{-1}\right)=\operatorname{Nrd}\left(\Pi_{2}\right)=2$. Since $\mathfrak{P}_{2}=\left\langle\Pi_{2}\right\rangle$ is the unique prime ideal over $2, x \Pi_{2} x^{-1} \in \mathfrak{P}_{2}$ and $x \gamma x^{-1} \in \Gamma_{\mathcal{O}}^{1}\left(\mathfrak{P}_{2}\right)$. It follows that the normalizer of $\Gamma_{\mathcal{O}}^{1}\left(\mathfrak{P}_{2}\right)$ is $N \Gamma_{\mathcal{O}}^{+}$. This leads to an exact sequence

$$
1 \longrightarrow \Gamma_{\mathcal{O}}^{1} / \Gamma_{\mathcal{O}}^{1}\left(\mathfrak{P}_{2}\right) \longrightarrow N \Gamma_{\mathcal{O}}^{+} / \Gamma_{\mathcal{O}}^{1}\left(\mathfrak{P}_{2}\right) \longrightarrow N \Gamma_{\mathcal{O}}^{+} / \Gamma_{\mathcal{O}}^{1} \longrightarrow 1
$$

which we can write abstractly as

$$
1 \longrightarrow \mathbb{Z} / 5 \mathbb{Z} \longrightarrow \operatorname{Aut}\left(X_{\Gamma_{\mathcal{O}}^{1}\left(\Pi_{2}\right)}\right) \longrightarrow \mathbb{Z} / 2 \mathbb{Z} \times \mathbb{Z} / 2 \mathbb{Z} \longrightarrow 1 .
$$

Let $\lambda \in \mathcal{O}^{1}$ satisfy $\lambda^{5}=-1$, that is, $\lambda$ gives rise to a 5 -torsion in $\Gamma_{\mathcal{O}}^{1}$. Then $\lambda$ satisfies the equation $\lambda^{2}-\frac{1+\sqrt{5}}{2} \lambda+1=0$ over $k$. We can assume that $\lambda$ generates $\Gamma_{\mathcal{O}}^{1} / \Gamma_{\mathcal{O}}^{1}\left(\mathfrak{P}_{2}\right)$. Let $g=\lambda+1$. The reduced norm of $g$ is $\operatorname{Nrd}(g)=(\lambda+1)(\bar{\lambda}+1)=$ $\operatorname{Nrd}(\lambda)+\operatorname{Trd}(\lambda)+1=2+\frac{1+\sqrt{5}}{2}=\frac{5+\sqrt{5}}{2}$, where $\operatorname{Trd}$ is the reduced trace and $x \mapsto \bar{x}$ is the standard involution of first kind on $B$. Since $\frac{5+\sqrt{5}}{2}$ is a totally positive generator of the prime ideal over 5,g defines an element of $N \Gamma_{\mathcal{O}}^{+}$(see (4-2)). On the other hand $g^{2}=(\lambda+1)^{2}=\lambda^{2}+2 \lambda+1=\left(\frac{1+\sqrt{5}}{2} \lambda-1\right)+2 \lambda+1=\left(\frac{5+\sqrt{5}}{2}\right) \lambda$. This shows that $g$ has order 10 in $N \Gamma_{\mathcal{O}}^{+}$and hence gives an element of order 10 in $N \Gamma_{\mathcal{O}}^{+} / \Gamma_{\mathcal{O}}^{1}\left(\mathfrak{P}_{2}\right)$. Moreover the image of $g$ in $N \Gamma_{\mathcal{O}}^{+} / \Gamma_{\mathcal{O}}^{1}$ is not trivial. Using the computer algebra system PARI, we can check that both ramified primes $\mathfrak{p}_{2}$ and $\mathfrak{p}_{5}$ are not split in $k(\sqrt{-2})$. This implies that $k(\sqrt{-2}) \subset B$ (see [Shavel 1978, Proposition 4.5]) and we can take $\sqrt{-2}$ as the generator $\Pi_{2}$ of $\mathfrak{P}_{2}$. Hence, $\Pi_{2}$, considered as an element of $N \Gamma_{\mathcal{O}}^{+}$, is of order 2 and the images of $g$ and $\Pi_{2}$ in $N \Gamma_{\mathcal{O}}^{+} / \Gamma_{\mathcal{O}}^{1}$ generate this group.

Lemma 4.14. Let $g$ and $\Pi_{2}$ be elements constructed above. Then in $N \Gamma_{\mathcal{O}}^{+}$we have the relation $\Pi_{2} g \Pi_{2}=g^{-1}$ modulo $\Gamma_{\mathcal{O}}^{1}\left(\mathfrak{P}_{2}\right)$.

Proof. The element $\Pi_{2}$ generates $\mathfrak{P}_{2}$. Consider $g$ and $\Pi_{2}$ as the elements of the localization $B_{\mathfrak{p}_{2}}$ of $B$ at $\mathfrak{p}_{2}$. This is a division quaternion algebra over $k_{\mathfrak{p}_{2}}$ and has a 
representation

$$
B_{\mathfrak{p}_{2}}=L_{\mathfrak{p}_{2}} \oplus \Pi_{2} L_{\mathfrak{p}_{2}},
$$

where $L_{\mathfrak{p}_{2}}$ is the unique unramified quadratic extension of $k_{\mathfrak{p}_{2}}$ (see [Vignéras 1980, p. 34]). For every $t \in L_{\mathfrak{p}_{2}}$ we have $t \Pi_{2}=\Pi_{2} \bar{t}$, where $\bar{t}$ is the Galois-conjugate of $t$ in $L_{\mathfrak{p}_{2}}$. The element $g$ lies in $k_{\mathfrak{p}_{2}}(\lambda)=k_{\mathfrak{p}_{2}}\left(\xi_{5}\right)$ which is an unramified quadratic extension of $k_{\mathfrak{p}_{2}}$, so $L_{\mathfrak{p}_{2}}=k_{\mathfrak{p}_{2}}(\lambda)$. Therefore $g \in L_{\mathfrak{p}_{2}}$ and $g \Pi_{2}=\Pi_{2} \bar{g}$. Because $g \bar{g}$ is in $k^{*}$ we have that $\bar{g}=g^{-1}$ considered as an element of $N \Gamma_{\mathcal{O}}^{+} \subset B_{\mathfrak{p}_{2}}^{*} / k_{\mathfrak{p}_{2}}^{*}$. This gives a relation

$$
\Pi_{2} g \Pi_{2}=g^{-1}
$$

in $N \Gamma_{\mathcal{O}}^{+}$, since $\Pi_{2}^{2}=1$ in $N \Gamma_{\mathcal{O}}^{+}$. Also, $g$ and $\Pi_{2} g \Pi_{2}=g^{-1}$ are not equal modulo $\Gamma_{\mathcal{O}}^{1}\left(\mathfrak{P}_{2}\right)$ because this would imply that $g^{2} \in \Gamma_{\mathcal{O}}^{1}\left(\mathfrak{P}_{2}\right)$. But as $\Gamma_{\mathcal{O}}^{1}\left(\mathfrak{P}_{2}\right)$ is torsion-free and $g^{2}$ is of finite order, this is impossible.

Proposition 4.15. With above notations we have

$$
\operatorname{Aut}\left(X_{\Gamma_{\mathcal{O}}^{1}\left(\mathfrak{P}_{2}\right)}\right) \cong \mathbb{D}_{10} .
$$

Proof. By the above discussion, $\operatorname{Aut}\left(X_{\Gamma_{\mathcal{O}}^{1}\left(\mathfrak{P}_{2}\right)}\right)$ is of order 20 and is generated by elements $g$ of order 10 and $\Pi_{2}$ of order 2 satisfying $\Pi_{2} g \Pi_{2}=g^{-1}$. The only group of order 20 with these relations is $\mathbb{D}_{10}$.

A fake quadric with automorphism group of order 8 . We consider $k=\mathbb{Q}(\sqrt{5})$ and $B=B\left(k, \mathfrak{p}_{2} \mathfrak{p}_{11}\right)$, the unique quaternion algebra ramified exactly at the primes $\mathfrak{p}_{2}$ and $\mathfrak{p}_{11}$. Since 2 is inert and 11 is decomposed in $k$, Shimizu's volume formula gives $c_{2}\left(X_{\Gamma_{\mathcal{O}}^{1}}\right)=\frac{4}{5 \cdot 12}(4-1)(11-1)=2$ as the value of the second Chern number of the quotient $X_{\Gamma_{\mathcal{O}}^{1}}$, where again $\Gamma_{\mathcal{O}}^{1}$ is the norm- 1 group of a maximal order in $B$. As before, results of [Shavel 1978] show that $\Gamma_{\mathcal{O}}^{1}$ contains only torsion elements of order 2 and no other torsions (Here, observe that 2 is split in $\mathbb{Q}(\sqrt{-15})$, hence, by [Shavel 1978, Theorem 4.8] there are no elements of order 3 in $\Gamma_{\mathcal{O}}^{1}$, and note that there are no elements of order 5 because $11 \equiv 1 \bmod 5$ which implies that $\mathfrak{p}_{11}$ is split in $k\left(\xi_{5}\right)$ ). Since $\mathfrak{p}_{11}$ is ramified in $B$, there is the unique prime ideal $\mathfrak{P}_{11}$ in $\mathcal{O}$ such that $\mathfrak{P}_{11}^{2}=\mathfrak{p}_{11} \mathcal{O}$. Consider the principal congruence subgroup

$$
\mathcal{O}^{1}\left(\mathfrak{P}_{11}\right)=\left\{x \in \mathcal{O}^{1} \mid x \equiv 1 \bmod \mathfrak{P}_{11}\right\}
$$

and $\Gamma_{\mathcal{O}}\left(\mathfrak{P}_{11}\right)$ its image in $\Gamma_{\mathcal{O}}^{1}$. It is a normal subgroup in $\Gamma_{\mathcal{O}}^{1}$. The quotient $\Gamma_{\mathcal{O}}^{1} / \Gamma_{\mathcal{O}}^{1}\left(\mathfrak{P}_{11}\right)$ is isomorphic to $\mathcal{O}^{1} / \pm \mathcal{O}^{1}\left(\mathfrak{P}_{11}\right)$ because $-1 \notin \mathcal{O}^{1}\left(\mathfrak{P}_{11}\right)$. In order to compute the latter quotient we change over to the localization at the prime $\mathfrak{p}_{11}$. Let

$$
B_{\mathfrak{p}_{11}}=B \otimes_{k} k_{\mathfrak{p}_{11}}=B \otimes_{k} \mathbb{Q}_{11} .
$$

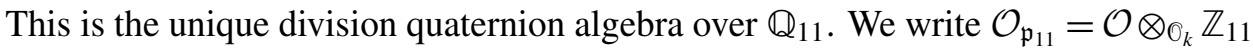


for its maximal order. As in the previous example let $\widehat{\mathfrak{P}}_{11}$ denote the prime ideal of $\mathcal{O}_{\mathfrak{p}_{11}}$. We have

$$
\mathcal{O}^{1} / \mathcal{O}^{1}\left(\mathfrak{P}_{11}\right) \cong \mathcal{O}_{\mathfrak{p}_{11}}^{1} / \mathcal{O}_{\mathfrak{p}_{11}}^{1}\left(\widehat{\mathfrak{P}}_{11}\right)
$$

by the strong approximation theorem. By Riehm's result [1970, Theorem 7],

$$
\mathcal{O}_{\mathfrak{p}_{11}}^{1} / \mathcal{O}_{\mathfrak{p}_{11}}^{1}\left(\widehat{\mathfrak{P}}_{11}\right) \cong \operatorname{ker}\left(\left(\mathcal{O}_{\mathfrak{p}_{11}} / \widehat{\mathfrak{P}}_{11}\right)^{*} \stackrel{\mathrm{Nr}}{\longrightarrow}\left(\mathcal{O}_{k_{\mathfrak{p}_{11}}} / \mathfrak{p}_{11}\right)^{*}\right) \cong \operatorname{ker}\left(\mathbb{F}_{121}^{*} \longrightarrow \mathbb{F}_{11}^{*}\right) .
$$

Since $\mathbb{F}_{121}=\mathbb{F}_{11}\left(\xi_{12}\right)$, where $\xi_{12}$ denotes a primitive twelfth root of unity we conclude that $\mathcal{O}_{\mathfrak{p}_{11}}^{1} / \mathcal{O}_{\mathfrak{p}_{11}}^{1}\left(\widehat{\mathfrak{P}}_{11}\right)$ is isomorphic to $\mu_{12}=\left\langle\xi_{12}\right\rangle$. Hence

$$
\Gamma_{\mathcal{O}}^{1} / \Gamma_{\mathcal{O}}^{1}\left(\mathfrak{P}_{11}\right) \cong \mathcal{O}_{\mathfrak{p}_{11}}^{1} / \pm \mathcal{O}_{\mathfrak{p}_{11}}^{1}\left(\widehat{\mathfrak{P}}_{11}\right) \cong \mu_{6}=\left\langle\xi_{6}\right\rangle
$$

Let us now define an intermediate group

$$
\Gamma=\left\{x \in \Gamma_{\mathcal{O}}^{1} \mid x \bmod \mathfrak{P}_{11} \in\left\langle\xi_{6}^{2}\right\rangle \subset \mu_{6}\right\} .
$$

$\Gamma<\Gamma_{\mathcal{O}}^{1}$ is a subgroup of index 2 , hence $c_{2}\left(X_{\Gamma}\right)=4$. Moreover, $\Gamma$ is torsion-free since it cannot contain elements of order 2. For if an order-two element $x$ is in $\Gamma$, then its image $x \bmod \mathfrak{P}_{11}$ in $\Gamma_{\mathcal{O}}^{1} / \Gamma_{\mathcal{O}}^{1}\left(\mathfrak{P}_{11}\right)$ lies in a cyclic group $\left\langle\xi_{6}^{2}\right\rangle$ of order three, hence it must be the identity. But this means that $x$ is in $\Gamma_{\mathcal{O}}^{1}\left(\mathfrak{P}_{11}\right)$. On the other hand $\Gamma_{\mathcal{O}}^{1}\left(\mathfrak{P}_{11}\right)$ is torsion-free because it embeds in a pro- 11 group $\mathcal{O}_{\mathfrak{p}_{11}}^{1}\left(\widehat{\mathfrak{P}}_{11}\right) / \pm 1$. This contradicts the assumption on $x$. All this shows that $X_{\Gamma}$ is a fake quadric.

Proposition 4.16. Let $N \Gamma_{\mathcal{O}}^{+}$be defined as in (4-1). Then $N \Gamma_{\mathcal{O}}^{+}$is the normalizer of $\Gamma$ and $N \Gamma_{\mathcal{O}}^{+} / \Gamma$ is isomorphic to $\mathbb{D}_{4}$.

Proof. As a subgroup of index 2 in $\Gamma_{\mathcal{O}}^{1}$ the group $\Gamma$ is normal in $\Gamma_{\mathcal{O}}^{1}$. On the other hand, for the same reason as in the previous example, $\Gamma_{\mathcal{O}}^{1}\left(\mathfrak{P}_{11}\right)$ as well as $\Gamma_{\mathcal{O}}^{1}$ is normal subgroup in $N \Gamma_{\mathcal{O}}^{+}$. This already implies that $\Gamma$ is normal in $N \Gamma_{\mathcal{O}}^{+}$because any conjugate of $\Gamma$ will be a subgroup between $\Gamma_{\mathcal{O}}^{1}\left(\mathfrak{P}_{11}\right)$ and $\Gamma_{\mathcal{O}}^{1}$ of index 2 in $\Gamma_{\mathcal{O}}^{1}$. There is only one such group, namely $\Gamma$, since $\Gamma_{\mathcal{O}}^{1} / \Gamma_{\mathcal{O}}^{1}\left(\mathfrak{P}_{11}\right) \cong \mathbb{Z} / 6 \mathbb{Z}$. Similar exact sequence as (4-3) now shows that $\operatorname{Aut}\left(X_{\Gamma}\right)$ is an extension of $\mathbb{Z} / 2 \mathbb{Z}$ by the Klein's four group. Since the 2-torsions in $\Gamma_{\mathcal{O}}^{1}$ come from embeddings of fourth roots of unity into $\mathcal{O}$ there is $\lambda \in \mathcal{O}^{1}$ such that $\lambda^{2}=-1$. Let $g=\lambda+1$. Then, as $\operatorname{Trd}(\lambda)=0$, we have $\operatorname{Nrd}(g)=(\lambda+1)(\bar{\lambda}+1)=2$ and also $g^{2}=(\lambda+1)^{2}=2 \lambda$ which implies that $g$ defines an element of order 4 in $N \Gamma_{\mathcal{O}}^{+}$and hence an element of order 4 in $N \Gamma_{\mathcal{O}}^{+} / \Gamma$. Moreover, the image of $g$ in $N \Gamma_{\mathcal{O}}^{+} / \Gamma_{\mathcal{O}}^{1}$ is not trivial. Since both prime divisors 2 and $\pi_{11}$ of the reduced discriminant do not split in $k\left(\sqrt{-\pi_{11}}\right)$ (as can be checked using PARI, for instance), the element $\Pi_{11}=\sqrt{-\pi_{11}}$ is in $B$ and moreover $\Pi_{11}$ defines an element of $N \Gamma_{\mathcal{O}}^{+}$of order 2 such that the images of $\Pi_{11}$ and $g$ in $N \Gamma_{\mathcal{O}}^{+} / \Gamma_{\mathcal{O}}^{1}$ generate this group. Same argument as in Lemma 4.14 gives a relation between $\Pi_{11}$ and $g$ : consider $\Pi_{11}$ as the generator of the prime ideal $\mathfrak{P}_{11}$. Locally, $B_{\mathfrak{p}_{11}}$ can be written as $B_{\mathfrak{p}_{11}}=L_{\mathfrak{p}_{11}} \oplus \Pi_{11} L_{\mathfrak{p}_{11}}$, where $L_{\mathfrak{p}_{11}}=k_{\mathfrak{p}_{11}}\left(\xi_{12}\right)$ is 
the unique unramified quadratic extension of $k_{\mathfrak{p}_{11}} \cong \mathbb{Q}_{11}$ with the multiplication rule $t \Pi_{11}=\Pi_{11} \bar{t}$ for all $t \in L_{\mathfrak{p}_{11}}$. The element $g$ is in $L_{\mathfrak{p}_{11}}$, namely $g=1+\xi_{12}^{3}$. Then $g \Pi_{11}=\Pi_{11} \bar{g}=\Pi_{11}\left(1+\bar{\xi}_{12}{ }^{3}\right)=\Pi_{11}\left(1+\xi_{12}^{9}\right)$. In $N \Gamma_{\mathcal{O}}^{+}$the relations $\bar{g}=g^{-1}$ and $\Pi_{11}^{2}=1$ hold, hence $\Pi_{11} g \Pi_{11}=g^{-1}$ in $N \Gamma_{\mathcal{O}}^{+}$. Also $g \neq g^{-1}$ modulo $\Gamma$, since otherwise $g^{2}$ would be in $\Gamma$ which is not possible because $g^{2}$ is torsion and $\Gamma$ torsion-free. $N \Gamma_{\mathcal{O}}^{+} / \Gamma$ is isomorphic to $\mathbb{D}_{4}$ which is the only group of order 8 generated by two elements $\Pi_{11}$ of order 2 and $g$ of order 4 with $h \neq g^{2}$ and $\Pi_{11} g \Pi_{11}=g^{-1}$.

Remark 4.17. Considering $k=\mathbb{Q}(\sqrt{13})$, the quaternion algebra $B=B\left(k, \mathfrak{p}_{2} \mathfrak{p}_{3}\right)$, and $\Gamma=\Gamma_{\mathcal{O}}^{1}\left(\mathfrak{P}_{3}\right)$, the arguments as in the examples before will show that $X_{\Gamma_{\mathcal{O}}^{1}\left(\mathfrak{P}_{3}\right)}$ is a fake quadric whose automorphism group is isomorphic to $\mathbb{D}_{4}$.

A fake quadric with automorphism group $\mathbb{D}_{6}$. This time we consider the quadratic field $k=\mathbb{Q}(\sqrt{2})$ and the quaternion algebra $B=B\left(k, \mathfrak{p}_{2} \mathfrak{p}_{3}\right)$. The norm-1 group $\Gamma_{\mathcal{O}}^{1}$ of a maximal order in $B$ contains torsion elements of order 3 , but no elements of order 2 , because $\mathfrak{p}_{3}$ is decomposed in $k(\sqrt{-1})$. The second Chern number of the quotient $X_{\Gamma_{\mathcal{O}}^{1}}$ is $c_{2}\left(X_{\Gamma_{\mathcal{O}}^{1}}\right)=(9-1) / 6=4 / 3$. Let $\Gamma_{\mathcal{O}}^{1}\left(\mathfrak{P}_{2}\right)$ be the principal congruence subgroup corresponding to the prime ideal $\mathfrak{P}_{2} \subset \mathcal{O}$, defined by the relation $\mathfrak{P}_{2}^{2}=\mathfrak{p}_{2} \mathcal{O}$. Again by Riehm's theorem and with arguments as in Section 4, $\Gamma_{\mathcal{O}}^{1}\left(\mathfrak{P}_{2}\right)$ is torsion-free normal subgroup in $\Gamma_{\mathcal{O}}^{1}$ of index 3 , hence $X_{\Gamma_{\mathcal{O}}}^{1}\left(\mathfrak{P}_{2}\right)$ is a fake quadric. The automorphism group $\operatorname{Aut}\left(X_{\Gamma_{\mathcal{O}}^{1}}^{1}\left(\mathfrak{P}_{2}\right)\right)$ is isomorphic to the factor group

$$
N \Gamma_{\mathcal{O}}^{+} / \Gamma_{\mathcal{O}}^{1}\left(\mathfrak{P}_{2}\right)
$$

which is an extension of $\Gamma_{\mathcal{O}}^{1} / \Gamma_{\mathcal{O}}^{1}\left(\mathfrak{P}_{2}\right) \cong \mathbb{Z} / 3 \mathbb{Z}$ by $N \Gamma_{\mathcal{O}}^{+} / \Gamma_{\mathcal{O}}^{1} \cong \mathbb{Z} / 2 \mathbb{Z} \times \mathbb{Z} / 2 \mathbb{Z}$.

Proposition 4.18. We have $\operatorname{Aut}\left(X_{\Gamma_{\mathcal{O}}^{1}\left(\mathfrak{P}_{2}\right)}\right) \cong \mathbb{D}_{6}$.

Proof. Let $\lambda \in \mathcal{O}^{1}$ be an element with $\lambda^{3}=-1$ and $g=\lambda+1$. Such $\lambda$ exists since $\Gamma_{\mathcal{O}}^{1}$ contains 3-torsions. We can take $\pm \lambda$ to be the generator of $\Gamma_{\mathcal{O}}^{1} / \Gamma_{\mathcal{O}}^{1}\left(\mathfrak{P}_{2}\right)$. Since $\operatorname{Trd}(\lambda)=1$, we have $\operatorname{Nrd}(g)=3$ which implies that $g$ defines an element in $N \Gamma_{\mathcal{O}}^{+}$. Additionally $g^{2}=\lambda^{2}+2 \lambda+1=3 \lambda$ which means that $g$ has order 6 considered as an element of $N \Gamma_{\mathcal{O}}^{+}$. The totally positive element $\pi_{2}=2+\sqrt{2} \in k$ generates $\mathfrak{p}_{2}$ and since neither $\pi_{3}=3$ nor $\pi_{2}$ are split in $k\left(\sqrt{-\pi_{2}}\right), \Pi_{2}=\sqrt{-\pi_{2}}$ lies in $B$ and defines an element in $N \Gamma_{\mathcal{O}}^{+}$of order 2 such that the classes of $g$ and $\Pi_{2}$ in $N \Gamma_{\mathcal{O}}^{+} / \Gamma_{\mathcal{O}}^{1}$ generate this group. In particular, $\Pi_{2}$ is a generator of $\mathfrak{P}_{2}$. Locally $B_{\mathfrak{p}_{2}}=L_{\mathfrak{p}_{2}} \oplus \Pi_{2} L_{\mathfrak{p}_{2}}$, where $L_{\mathfrak{p}_{2}}=\mathbb{Q}_{2}\left(\xi_{6}\right)$ is the unramified quadratic extension of $k_{\mathfrak{p}_{2}} \cong \mathbb{Q}_{2}$. As in previous examples, $g$ lies in $L_{\mathfrak{p}_{2}}$ and $\Pi_{2} g \Pi_{2}=\bar{g}=g^{-1}$ in $N \Gamma_{\mathcal{O}}^{+}$. This gives a relation $\Pi_{2} g \Pi_{2}=g^{-1}$ in $N \Gamma_{\mathcal{O}}^{+} / \Gamma_{\mathcal{O}}^{1}\left(\mathfrak{P}_{2}\right)$. As $\Pi_{2}$ is not a power of $g$, the finite group generated by $g$ and $\Pi_{2}$ is isomorphic to $\mathbb{D}_{6}$. 
Automorphism groups of order 16 and 24. There are more examples of quaternionic fake quadrics with a nontrivial automorphism group. For instance, all examples in Shavel's paper have $\mathbb{Z} / 2 \mathbb{Z}$ or $(\mathbb{Z} / 2 \mathbb{Z})^{2}$ as the full group of automorphisms. As in previous examples we show

Proposition 4.19. Let $B\left(\mathbb{Q}(\sqrt{2}), \mathfrak{p}_{2}, \mathfrak{p}_{7}\right)$ be the indefinite quaternion algebra over $k=\mathbb{Q}(\sqrt{2})$ with reduced discriminant $d_{B}=\mathfrak{p}_{2} \mathfrak{p}_{7}$ and $\Gamma_{\mathcal{O}}^{1}\left(\mathfrak{P}_{7}\right)$ the congruence subgroup in $\Gamma_{\mathcal{O}}^{1}$ corresponding to a maximal order $\mathcal{O}$ in $B$ with respect to the prime ideal $\mathfrak{P}_{7}$ of $\mathcal{O}$ lying over the ramified prime $\mathfrak{p}_{7}$. Then $X_{\Gamma_{\mathcal{O}}^{1}\left(\mathfrak{P}_{7}\right)}$ is a fake quadric with the automorphism group $\operatorname{Aut}\left(X_{\Gamma_{\mathcal{O}}^{1}\left(\mathfrak{P}_{7}\right)}\right) \cong \mathbb{D}_{8}$.

Proof. The proof goes along the same lines as in the examples before. By Riehm's Theorem, $\Gamma_{\mathcal{O}}^{1} / \Gamma_{\mathcal{O}}^{1}\left(\mathfrak{P}_{7}\right) \cong \mathbb{Z} / 4 \mathbb{Z}$ and we obtain $c_{2}\left(X_{\Gamma_{\mathcal{O}}^{1}\left(\mathfrak{P}_{7}\right)}\right)=4$ by Shimizu's formula. By Shavel's criterion for the existence of torsions, we find that the maximal order $\mathcal{O}$ contains a primitive eighth root of unity $\lambda$ which leads to an element of order 4 in $\Gamma_{\mathcal{O}}^{1}$. We can take $\lambda$ as a generator of this quotient. As in the examples before take $g=1+\lambda$. Then, as $\lambda$ satisfies $\lambda^{2}-\sqrt{2} \lambda+1=0$ over $k$, $\operatorname{Nrd}(g)=\operatorname{Nrd}(\lambda+1)=2+\sqrt{2}$, hence $g$ defines an element in $N \Gamma_{\mathcal{O}}^{+}$. We have $g^{2}=\lambda^{2}+2 \lambda+1=\sqrt{2} \lambda+2 \lambda=(2+\sqrt{2}) \lambda$. Hence, $g$ is an element of order 8 in $N \Gamma_{\mathcal{O}}^{+}$and its image in $N \Gamma_{\mathcal{O}}^{+} / \Gamma_{\mathcal{O}}^{1}$ is not trivial. The rational prime 7 is split in $k$, so there are two possible choices of $\mathfrak{p}_{7}$. Fix a prime $\mathfrak{p}_{7}=\left\langle\pi_{7}\right\rangle\left(\pi_{7}=3+\sqrt{2}\right.$ say). Both $\pi_{7}$ as well as $\pi_{2}$ are ramified in $k\left(\sqrt{-\pi_{7}}\right)$, hence $\sqrt{-\pi_{7}} \in B$ defines an element $\Pi_{7} \in B$ which defines an order-2 element in $N \Gamma_{\mathcal{O}}^{+}$. As in the previous examples we have $\Pi_{7} g \Pi_{7}=\bar{g}$ because locally in $B_{\mathfrak{p}_{7}}, \Pi_{7}=\sqrt{-\pi_{7}}$ generates the unique prime ideal of the maximal order $\mathcal{O}_{\mathfrak{p}_{7}}$ and $g$ lies in the unramified quadratic extension $L_{\mathfrak{p}_{7}}=\mathbb{Q}_{7}\left(\xi_{8}\right)$. This gives a relation $\Pi_{7} g \Pi_{7}=g^{-1}$ in $N \Gamma_{\mathcal{O}}^{+} / \Gamma_{\mathcal{O}}^{1}\left(\mathfrak{P}_{7}\right)$. Also $\Pi_{7}$ is not a power of $g$ modulo $\Gamma_{\mathcal{O}}^{1}\left(\mathfrak{P}_{7}\right)$ since the reduced norms of $\Pi_{7}$ and $g$ are different primes. The only group of order 16 with these relations is $\mathbb{D}_{8}$.

Let us finally sketch the construction of a fake quadric with an automorphism group of order 24 .

Proposition 4.20. Let $B\left(\mathbb{Q}(\sqrt{3}), \mathfrak{p}_{2}, \mathfrak{p}_{3}\right)$ be the indefinite quaternion algebra over $k=\mathbb{Q}(\sqrt{3})$ ramified over the prime ideals $\mathfrak{p}_{2}$ and $\mathfrak{p}_{3}$ and let $\Gamma_{\mathcal{O}}^{1}\left(\mathfrak{P}_{2} \mathfrak{P}_{3}\right) \triangleleft \Gamma_{\mathcal{O}}^{1}$ be the principal congruence subgroup with respect to the principal ideal $\mathfrak{P}_{2} \mathfrak{P}_{3}$ of a maximal order $\mathcal{O} \subset B$ lying over $\mathfrak{p}_{2} \mathfrak{p}_{3}$. Then $X_{\Gamma_{\mathcal{O}}^{1}\left(\mathfrak{P}_{2} \mathfrak{P}_{3}\right)}$ is a fake quadric with $\left|\operatorname{Aut}\left(X_{\Gamma_{\mathcal{O}}^{1}\left(\mathfrak{P}_{2} \mathfrak{P}_{3}\right)}\right)\right|=24$. The automorphism group $\operatorname{Aut}\left(X_{\Gamma_{\mathcal{O}}^{1}\left(\mathfrak{P}_{2} \mathfrak{P}_{3}\right)}\right)$ contains a cyclic subgroup of order 12.

Remark 4.21. The full automorphism group in this case has order 24. To our knowledge, this is the largest known automorphism group of a fake quadric. The precise abstract group structure of $\operatorname{Aut}\left(X_{\Gamma_{\mathcal{O}}^{1}\left(\mathfrak{P}_{2} \mathfrak{F}_{3}\right)}\right)$ is not known to us, since the local method, used in previous examples does not apply directly in this case. 
Proof. That $X_{\Gamma_{\mathcal{O}}^{1}\left(\mathfrak{P}_{2} \mathfrak{P}_{3}\right)}$ has the correct numerical invariants follows again from Riehm's Theorem, Shimizu's formula and the observation that for the index we have $\left[\Gamma_{\mathcal{O}}^{1}: \Gamma_{\mathcal{O}}^{1}\left(\mathfrak{P}_{2} \mathfrak{P}_{3}\right)\right]=\left[\Gamma_{\mathcal{O}}^{1}: \Gamma_{\mathcal{O}}^{1}\left(\mathfrak{P}_{2}\right)\right]\left[\Gamma_{\mathcal{O}}^{1}: \Gamma_{\mathcal{O}}^{1}\left(\mathfrak{P}_{3}\right)\right]$. By Shavel's criterion, $B$ contains $k\left(\xi_{12}\right)$ where $\xi_{12}$ is a primitive twelfth root of unity, hence there is an element $\lambda \in \mathcal{O}$ with $\lambda^{6}=-1$. To show that $\Gamma_{\mathcal{O}}^{1}\left(\mathfrak{P}_{2} \mathfrak{P}_{3}\right)$ is torsion-free we have to exclude the existence of 6-torsions in $\Gamma_{\mathcal{O}}^{1}\left(\mathfrak{P}_{2} \mathfrak{P}_{3}\right)$. But since the reduced trace of $\lambda$ is $\pm \sqrt{3}$ which is not congruent 2 modulo $\mathfrak{p}_{2} \mathfrak{p}_{3}, \lambda$ is not contained in $\Gamma_{\mathcal{O}}^{1}\left(\mathfrak{P}_{2} \mathfrak{P}_{3}\right)$. The element $g=\lambda+1$ has norm $\operatorname{Nrd}(g)=2+\sqrt{3}$ which is a totally positive unit of $\mathrm{O}_{k}$ unit, hence $g$ lies in $\Gamma_{\mathcal{O}}^{+}=\mathcal{O}^{+} / \mathcal{O}_{k}^{*}$, where $\mathcal{O}^{+}$denotes the group of all units whose reduced norm is totally positive. The group $\Gamma_{\mathcal{O}}^{+}$is an index-2-extension of $\Gamma_{\mathcal{O}}^{1}$ since the fundamental unit $2+\sqrt{3}$ is totally positive. Also $g^{2}=(2+\sqrt{3}) \lambda$ which shows that $g$ has order 12 in $\Gamma_{\mathcal{O}}^{+} \triangleleft N \Gamma_{\mathcal{O}}^{+}$. The image of $g$ in $N \Gamma_{\mathcal{O}}^{+} / \Gamma_{\mathcal{O}}^{1}$ is not trivial and the discussion in [Shavel 1978, pp. 223-224] shows that $N \Gamma_{\mathcal{O}}^{+} / \Gamma_{\mathcal{O}}^{*}$ is generated by the class of an element $\Pi \in N \Gamma_{\mathcal{O}}^{+}$with $\operatorname{Nrd}(\Pi)=\pi_{2} \pi_{3}$ where $\mathfrak{p}_{2}=\left\langle\pi_{2}\right\rangle, \mathfrak{p}_{3}=\left\langle\pi_{3}\right\rangle$ (note that the generators $\pi_{2}$ and $\pi_{3}$ cannot be chosen totally positive). Therefore, $\operatorname{Aut}\left(X_{\left.\Gamma_{\mathcal{O}}^{1}\left(\mathfrak{P}_{2} \mathfrak{P}_{3}\right)\right)}\right)$ is of order 24 and is an extension of $\mathbb{Z} / 6 \mathbb{Z}$ by the Klein's four group.

\section{Computations of the quotient surfaces}

Let $S$ be a quaternionic fake quadric, $G$ a group of automorphisms of $S, S / G$ the quotient surface and let $\pi: Z \rightarrow S / G$ be the minimal desingularization map.

Let us first study the case where $G$ is generated by an involution $\sigma$.

Proposition 5.22. An involution $\sigma$ has 4 fixed points. The invariants of $Z$ are

$$
K_{Z}^{2}=4, \quad c_{2}=8, \quad q=p_{g}=0, \quad h^{1,1}=5 .
$$

The surface $Z$ is minimal of general type.

Proof. By Lefschetz's formula (Proposition 2.6), $1=\sum_{s=\sigma(s)} \frac{1}{4}$, therefore $\sigma$ has 4 fixed points. Their images in $S / \sigma$ are $4 A_{1}$ singularities, resolved by $4(-2)$-curves on $Z$. The invariants of $Z$ are easy to compute.

The surface $Z$ is of general type and is minimal because $K_{Z}$ is the pullback of the nef divisor $K_{S / G}$.

Proposition 5.23. Let $G=\langle\sigma\rangle \cong \mathbb{Z} / 3 \mathbb{Z}$. The singularities of the quotient surface $S / G$ are $2 A_{3,1}+2 A_{3,2}$. The resolution $Z$ has general type, and

$$
K_{Z}^{2}=2, \quad c_{2}=10, \quad q=p_{g}=0 .
$$

Proof. We use the notations of Zhang's formula (Proposition 2.7). In this case this formula gives $r_{1}+r_{2}=4$. An $A_{3,1}$ singularity is resolved by a (-3)-curve, and 
we have

$$
K_{Z}^{2}=\frac{8}{3}-\frac{r_{1}}{3}
$$

Therefore $r_{1}=2$ and $r_{2}=2$. The singularities of $S / G$ are $2 A_{3,1}+2 A_{3,2}$. Moreover, as $q=p_{g}=0$, we have $c_{2}=10 . Z$ is of general type by Lemma 2.11 .

Proposition 5.24. There is no quaternionic fake quadric $S$ with $G=(\mathbb{Z} / 3 \mathbb{Z})^{2} \subset$ Aut $S$.

Proof. Let $\sigma_{1}, \sigma_{2}$ be the two generating elements of $G$. Let $p$ be one of the 4 fixed points of $\sigma_{1}$ (see Proposition 5.23). Since $\sigma_{1}$ and $\sigma_{2}$ commute, the set of fixed points of $\sigma_{1}$ is sent to itself by $\sigma_{2}$, indeed there are two orbits of 2 elements because of the different local type of the action of $\sigma_{1}$. Now $\sigma_{2}$ has order three, hence it acts trivially on these 2 orbits and the conclusion is that there are 4 fixed points for the action of the whole group $G$. The faithful action of $G$ on the tangent space of $p$ can be diagonalized, hence there are elements with one eigenvalue equal to 1 , contradicting Lemma 2.8 and Theorem 3.12.

Proposition 5.25. Let $G=\mathbb{Z} / 4 \mathbb{Z}$. The singularities of the quotient $S / G$ are $2 A_{4,1}+$ $2 A_{4,3}$ or $A_{1}+2 A_{4,3}$. The invariants of the resolution $Z$ are

$$
K_{Z}^{2}=0, \quad c_{2}=12, \quad q=p_{g}=0
$$

in the first case, and in the second case $Z$ is minimal and satisfies

$$
K_{Z}^{2}=2, \quad c_{2}=10, \quad q=p_{g}=0 .
$$

Remark 5.26. Proposition 5.34 gives an example of the first case.

Proof. Let $s$ be a fixed point of an order 4 automorphism $\sigma$ acting on $S$. As the involution $\sigma^{2}$ has only isolated fixed points, the eigenvalues of $\sigma$ acting on $T_{S, s}$ cannot be $(i,-1)$ or $(-i,-1)$. Let $a, b, c$ be the number of fixed points such that the eigenvalues of $\sigma$ are $(i, i),(-i,-i)$ and $(i,-i)$ respectively. The Lefschetz holomorphic fixed point formula implies

$$
-\frac{a}{2 i}+\frac{b}{2 i}+\frac{c}{2}=1 \quad \text { and } \quad a+b+c=4 \text { or } 2,
$$

thus there are two cases:

(1) $a=b=1$ and $c=2$. The singularities of $S / G$ are $2 A_{4,1}+2 A_{4,3}$.

(2) $a=b=0$ and $c=2$. In this case, the singularities of $S / G$ are $A_{1}+2 A_{4,3}$ because $\sigma^{2}$ has 4 fixed points. 
An $A_{4,1}$ singularity is resolved by a (-4)-curve $C_{k}$ and an $A_{4,3}$ singularity is resolved by a chain of three $(-2)$-curves and we have

$$
K_{Z}=\pi^{*} K_{S / \sigma}-\sum_{k=1}^{k=2} \frac{1}{2} C_{k},
$$

thus $K_{Z}^{2}=\frac{8}{4}-2=0$ in the first case. Additionally,

$$
e(S / \sigma)=\frac{1}{4}(4+(4-1) 4)=4,
$$

thus $c_{2}(Z)=4+8=12$. The invariants in the second case are computed in a similar way.

Proposition 5.27. Let $G=\mathbb{Z} / 5 \mathbb{Z}$. The singularities of $S / G$ are $4 A_{5,2}$ or $A_{5,1}+$ $2 A_{5,2}+A_{5,4}$ or $2 A_{5,1}+2 A_{5,4}$. The invariants of the surface $Z$ are, respectively,

$$
\begin{array}{cccc}
K_{Z}^{2}=0 \\
c_{2}=12
\end{array} \quad \text { or } \quad \begin{gathered}
K_{Z}^{2}=-1 \\
c_{2}=13
\end{gathered} \quad \text { or } \quad \begin{gathered}
K_{Z}^{2}=-2 \\
c_{2}=14,
\end{gathered}
$$

and in any case $q=p_{g}=0$.

Remark 5.28. (1) In Proposition 5.31 below, we give an example of a surface such that the quotient by an order 5 automorphism has $2 A_{5,1}+2 A_{5,4}$ singularities.

(2) By the same kind of arguments as for $(\mathbb{Z} / 3 \mathbb{Z})^{2}$ (see Proposition 5.24), there is no fake quadric $S$ with $(\mathbb{Z} / 5 \mathbb{Z})^{2} \subset$ Aut $S$.

Proof. Using the notations of Proposition 2.7, the number of fixed points $r_{1}+r_{2}+$ $r_{3}+r_{4}$ equals 4 . As $e(S / \sigma)=\frac{1}{5}(4+(5-1) 4)=4$, Zhang's formula yields

$$
\left(a_{1}, \ldots, a_{4}\right)=\left(0, \frac{1}{4}, \frac{1}{4}, \frac{1}{2}\right) \text {, }
$$

with

$$
\sum 4 a_{i} r_{i}=r_{2}+r_{3}+2 r_{4}=4
$$

Thus $r_{1}=r_{4}$. Therefore the possibilities for $\left(r_{1}, r_{2}, r_{3}, r_{4}\right)$ are $(0, i, j, 0)$ with $i+j=4$, or $(1, i, j, 1)$ with $i+j=2$, or $(2,0,0,2)$. The singularities on the quotient are, respectively,

$$
4 A_{5,2} \quad \text { or } A_{5,1}+2 A_{5,2}+A_{5,4} \quad \text { or } \quad 2 A_{5,1}+2 A_{4} .
$$

A singularity $A_{5, i}(i=1, \ldots, 4)$ contributes (respectively)

$$
-\frac{9}{5},-\frac{2}{5},-\frac{2}{5}, 0
$$

to $K_{Z}^{2}$. Thus the self-intersection number is

$$
K_{Z}^{2}=\frac{1}{5}\left(8-9 r_{1}-2\left(r_{2}+r_{3}\right)\right),
$$


and according to the possible tuples $\left(r_{1}, \ldots, r_{4}\right)$ as above: $K_{Z}^{2}=0$, or $K_{Z}^{2}=-1$, or $K_{Z}^{2}=-2$. As $e(S / G)=4$, we get $c_{2}=12,13$, or 14 according to the three possible singular loci.

Let us justify our computation of $K_{Z}^{2}$. An $A_{5,1}$-singularity is resolved by a $(-5)$-curve $C_{5}$, thus we have to add $-\frac{3}{5} C_{5}$ to the canonical divisor. This contributes $\left(-\frac{3}{5} C_{5}\right)^{2}=-\frac{9}{5}$ to $K_{Z}^{2}$. On the other hand, an $A_{5,2}$-singularity is resolved by a chain of two curves $C_{2}, C_{3}$ with $C_{k}^{2}=-k$. We have to add $-\frac{2}{5} C_{3}-\frac{1}{5} C_{2}$ to $\pi^{*} K_{S / G}$, and the contribution to $K_{Z}^{2}$ is

$$
\left(\frac{2}{5} C_{3}+\frac{1}{5} C_{2}\right)^{2}=-\frac{2}{5} .
$$

Finally, note that $A_{5,3}=A_{5,2}$, and the $A_{5,4}$-singularity does not contribute to $K_{Z}^{2}$.

Proposition 5.29. If $G=\mathbb{Z} / 6 \mathbb{Z}$, then $S / G$ has singularities $2 A_{6,1}+2 A_{6,5}$. The minimal resolution $Z$ has invariants

$$
K_{Z}^{2}=-4, \quad c_{2}=16, \quad q=p_{g}=0 .
$$

Proof. Let $s$ be a fixed point of an order 6 automorphism $\sigma$. Let $\alpha$ be a primitive third root of unity. By Lemma 2.8, the action of $\sigma$ on $T_{S, s}$ has eigenvalues $\left(-\alpha,(-\alpha)^{a}\right)$ or $\left(-\alpha^{2},\left(-\alpha^{2}\right)^{a}\right)$ with $a=1$ or 5 . Let $r_{1}, r_{2}$ and $r_{3}$ be respectively the number of fixed points of $\sigma$ with eigenvalues $(-\alpha,-\alpha),\left(-\alpha^{2},-\alpha^{2}\right)$ and $\left(-\alpha,-\alpha^{5}\right)$. Lefschetz fixed point formula (Proposition 2.6) implies the relation

$$
\frac{r_{1}}{(1+\alpha)^{2}}+\frac{r_{2}}{\left(1+\alpha^{2}\right)^{2}}+r_{3}=1,
$$

therefore $r_{1}=r_{2}$ and $-r_{1}+r_{3}=1$. By Corollary $2.5, \sigma$ has 2 or 4 fixed points. The only possibility for $\left(r_{1}, r_{3}\right)$ is therefore $(1,2)$. The singularities are $2 A_{6,1}+2 A_{6,5}$ and the minimal resolution $Z$ of $S / \sigma$ has $K_{Z}^{2}=\frac{8}{6}-2 \cdot \frac{8}{3}=-4$. Moreover $e(Z)=$ $\frac{1}{6}(4+5 \cdot 4)+2+2 \cdot 5=16$.

Let us study the case $G=\mathbb{Z} / 8 \mathbb{Z}$.

Proposition 5.30. Let $\sigma$ be an order 8 element acting on $S$. The singularities of $S / \sigma$ are $2 A_{8,3}+2 A_{8,5}$. The resolution $Z$ of the quotient surface is a surface with

$$
K_{Z}^{2}=-2, \quad c_{2}(Z)=14, \quad q=p_{g}=0 .
$$

Proof. Let $p$ be a fixed point of $\sigma$ and let $\xi_{(p)}$ be a primitive eighth root of unity such that $\sigma$ acts on $T_{S, p}$ with eigenvalues $\xi_{(p)}$ and $\xi_{(p)}^{q_{p}}$ for $q_{p} \in\{0, \ldots, 7\}$. There are no reflections, so we have $\xi_{(p)}^{j} \neq 1$ and $\xi_{(p)}^{j q_{p}} \neq 1$ for $j=1, \ldots, 7$, thus $q_{p}$ is prime to 2: $q_{p} \in\{1,3,5,7\}$. Let $a_{1}, a_{3}, a_{5}$ and $a_{7}$ be the number of fixed points $p$ with $q_{p}=1,3,5$ or 7 respectively. We have $\sum a_{i}=2$ or 4 . By summing over the powers $\sigma^{k}$ for $k=1, \ldots, 7$ in the formula of the holomorphic Lefschetz theorem, 
we get

$$
7=\sum_{p \in S^{\sigma}} \sum_{k=1}^{k=7} \frac{1}{\operatorname{det}\left(1-d \sigma^{k} \mid T_{S, p}\right)}
$$

and thus

$$
7=\sum_{u=0}^{u=3} \sum_{k=1}^{k=7} \frac{a_{2 u+1}}{\left(1-\xi^{k}\right)\left(1-\xi^{k(2 u+1)}\right)}=\frac{7}{4} a_{1}+\frac{5}{4} a_{3}+\frac{9}{4} a_{5}+\frac{21}{4} a_{7} .
$$

The possibilities for $\left(a_{1}, \ldots, a_{4}\right)$ are $(4,0,0,0),(2,1,1,0),(1,0,0,1)$ and $(0,2,2,0)$.

For $t^{2}$ of order 4 , we have seen that the singularities of $S / \sigma^{2}$ are $2 A_{4,1}+2 A_{4,3}$ or $A_{1}+2 A_{4,3}$. Thus the only possibility for $\left(a_{1}, \ldots, a_{4}\right)$ is $(0,2,2,0)$, and the singularities of $S / \sigma$ are $2 A_{8,3}+2 A_{8,5}$. The Euler number of $S / \sigma$ is

$$
e(S / \sigma)=\frac{1}{8}(4+7 \cdot 4)=4 \text {. }
$$

Since $\frac{8}{3}=3-\frac{1}{3}$ and $\frac{8}{5}=2-\frac{1}{3-\frac{1}{2}}$ we get

$$
e(Z)=4+2 \cdot 2+2 \cdot 3=14 \text {. }
$$

It is easy to check that a singularity $A_{8,3}$ decreases $K_{Z}^{2}$ by 1 and a singularity $A_{8,5}$ decreases $K_{Z}^{2}$ by $\frac{1}{2}$, thus we obtain $K_{Z}^{2}=\frac{8}{8}-2 \cdot 1-2 \cdot \frac{1}{2}=-2$.

Proposition 5.31. Let $S$ be a fake quadric with $G=\mathbb{Z} / 10 \mathbb{Z} \subset \operatorname{Aut}(S)$. The singularities of the quotient surface $S / G$ are $2 A_{10,1}+2 A_{10,9}$. The resolution $Z$ has the invariants

$$
K^{2}=-12, \quad c_{2}=24, \quad q=p_{g}=0 .
$$

Proof. Let $\sigma$ be an automorphism of order 10 acting on $S$. It has 2 or 4 fixed points. As the involution $\sigma^{5}$ has 4 fixed points, $\sigma$ cannot have 2 fixed points. Therefore

$$
e(S / G)=\frac{1}{10}(4+(10-1) 4)=4 .
$$

Let $\xi$ be a primitive fifth root of unity and $p$ a fixed point. There exist $a=a(p)$ and $b=b(p)$ integers invertible mod 5 such that the action of $\sigma$ on $T_{S, p}$ has eigenvalues $\left(-\xi^{a},-\xi^{b a}\right)$. The Lefschetz holomorphic fixed point formula yields

$$
1=\sum_{p \in S^{\sigma}} \frac{1}{\left(1+\xi^{a}\right)\left(1+\xi^{a b}\right)} .
$$

For $b=1,2,3,4$, the sum

$$
c(b)=\sum_{a=1}^{a=4} \frac{1}{\left(1+\xi^{a}\right)\left(1+\xi^{a b}\right)}
$$


is equal to $-4,1,1,6$, respectively. Recall again that $A_{10,3}=A_{10,7}$. For $k \in\{1,3,9\}$, let $r_{k}$ be the number of points in $S^{\sigma}$ giving an $A_{10, k}$ singularity. The Lefschetz fixed point formula gives

$$
4=-4 r_{1}+r_{3}+6 r_{9} .
$$

Taking care of the relation $r_{1}+r_{3}+r_{9}=4$, we have the following possibilities for $\left(r_{1}, r_{3}, r_{9}\right):(0,4,0),(1,2,1)$ and $(2,0,2)$.

The resolution of an $A_{10,3}$-singularity is a chain of 3 curves $C_{2}, C_{2}^{\prime}, C_{4}$ with intersection numbers $(-2)-(-2)-(-4)$. We have to add $-\frac{1}{5}\left(C_{2}+C_{2}^{\prime}+C_{4}\right)$ to $\pi^{*} K_{S / G}$. Each singularity contributes $\left(-\frac{1}{5}\left(C_{2}+C_{2}^{\prime}+C_{4}\right)\right)^{2}=-\frac{6}{5}$ to $K_{Z}^{2}$.

Similarly, the resolution of an $A_{10,1}$-singularity is a (-10)-curve $C_{10}$. An $A_{10,1^{-}}$ singularity decreases $K_{S / G}^{2}$ by $\left(-\frac{8}{10} C_{10}\right)^{2}=-\frac{32}{5}$.

When the singularities of $S / G$ are respectively $4 A_{10,3}, A_{10,1}+2 A_{10,3}+A_{10,9}$ and $2 A_{10,1}+2 A_{10,9}$, we have: $K_{Z}^{2}=\frac{8}{10}-4 \cdot \frac{6}{5}=-4, K_{Z}^{2}=\frac{8}{10}-\frac{32}{5}-2 \cdot \frac{6}{5}-0=-8$ and $K_{Z}^{2}=\frac{8}{10}-2 \cdot \frac{32}{5}=-12$, respectively. The Euler number of $Z$ is respectively $4+4 \cdot 2=12,4+1+2 \cdot 2+9=18$ and $4+2+2 \cdot 9=24$. Only the last case is possible because 12 has to divide $K_{Z}^{2}+e(Z)$.

Proposition 5.32. Let $G=(\mathbb{Z} / 2 \mathbb{Z})^{2}$. The quotient surface $S / G$ contains $6 A_{1}$ singularities. The surface $Z$ is minimal of general type and has the invariants

$$
K_{Z}^{2}=2, \quad c_{2}=10, \quad q=p_{g}=0 .
$$

Proof. A faithful representation of $G$ on a 2-dimensional space contains reflections, therefore by Lemma 2.8, there are no points fixed by the whole $G$. The group $G$ contains 3 involutions. Each of these involutions has 4 isolated fixed points whose image in $X$ are $2 A_{1}$ singularities. Thus there are $6 A_{1}$ singularities on $S / G$ and we have

$$
e(Z)=e(S / G)+6=\frac{1}{4}(4+12)+6=10 .
$$

Moreover, $K_{Z}=\pi^{*} K_{S / G}$ is nef and $K_{S / G}^{2}=K_{S}^{2} / 4=2$. By Lemma 2.10, we have $q=p_{g}=0$.

Remark 5.33. (a) Fabrizio Catanese and Miles Reid pointed out to us that a minimal surface of general type with $c_{1}^{2}=2 c_{2}=8, p_{g}=0$ and automorphism group containing $G=(\mathbb{Z} / 2 \mathbb{Z})^{3}$ such that each involution has only isolated points must deform, therefore $(\mathbb{Z} / 2 \mathbb{Z})^{3}$ cannot be a subgroup of the automorphism group of a quaternionic fake quadric, which is a rigid surface. The complete argument is as follows: The minimal resolution $Z$ of the quotient $Y=X / G$ of a fake quadric $X$ by $G$ would be a numerical Godeaux surface, that is, a surface with $c_{1}^{2}=1$ and with the maximal number of nodal curves, being equal to 7 . We do not know whether such a surface exists, but from coding theory one would have a covering $S \rightarrow Y$ with group $G$ ramified only over the nodes. 
The covering surface $S$ would have $c_{1}^{2}=8, p_{g}=0$. However, the Kuranishi family of deformations for the surface $Z$ has dimension greater or equal to the expected dimension, which is $8=10 \chi-2 c_{1}^{2}$, and the 7 nodes impose at most 1 condition each, because of the morphism of the global deformation space to the local deformation space of the singularities. Therefore this family of 7-nodal numerical Godeaux surfaces would have 1 modulus, and therefore also the above surfaces $S$ would vary in moduli. However, quotients of the bidisk by an irreducible subgroup are rigid, for instance, by a theorem of Jost and Yau.

(b) For $G=\mathbb{Z} / 4 \mathbb{Z} \times \mathbb{Z} / 2 \mathbb{Z}$, the quotient surface $S / G$ has singularities $2 A_{1}+2 A_{3}$ and the desingularization $Z$ has invariants $K_{Z}^{2}=1, c_{2}=11, q=p_{g}=0$. We do not know if a fake quadric $S$ with such automorphism subgroup exists.

Proposition 5.34. Let $G=\mathbb{D}_{4}$ acting on the fake quadric $S$. The singularities of $S / G$ are $4 A_{1}+A_{4,3}+A_{4,1}$. The resolution $Z$ of the quotient surface has invariants

$$
K_{Z}^{2}=0, \quad c_{2}(Z)=12, \quad q=p_{g}=0 .
$$

The elements of order 4 in $\mathbb{D}_{4}$ have 4 fixed points.

Proof. Let $t$ and $a$ be the generators of $\mathbb{D}_{4}$ such that $t^{4}=1, a^{2}=1$ and $a t=t^{3} a$. The elements of order 4 are $t$ and $t^{3}$. The elements of order 2 are $a, t a, t^{2} a, t^{3} a$ and $t^{2}$.

There cannot be a point of $S$ that is fixed by the whole group $G$ because any faithful 2-dimensional representation of $G$ contains a reflection $(x, y) \rightarrow(x,-y)$ and thus such a point would lie on a curve fixed by an involution, but an automorphism of $S$ has only isolated fixed points.

First case: Suppose that $t$ has 4 fixed points, $\operatorname{Fix}(t)=\left\{p_{1}, a p_{1}, p_{2}, a p_{2}\right\}$. The Euler number of $S / G$ is

$$
e(S / G)=\frac{1}{8}(4+(2-1)(4 \cdot 4)+(4-1) 4)=4 .
$$

The singularities on $S / G$ are $4 A_{1}+A_{4,3}+A_{4,1}$ and therefore

$$
e(Z)=4+4+3+1=12 \text {. }
$$

Moreover $K_{Z}^{2}=\frac{8}{8}+\left(-\frac{1}{2}\right)^{2}(-4)=0$.

Second case: Suppose that $t$ has 2 fixed points, $\operatorname{Fix}(t)=\left\{p_{1}, a p_{1}\right\}$. The Euler number of $e(S / G)$ would be

$$
\frac{1}{8}(4+(2-1) 18+(4-1) 2)=\frac{7}{2},
$$

but this is not an integer. 
Proposition 5.35. Suppose that the dihedral group $\mathbb{D}_{8}$ of order 16 acts on fake quadric $S$. The singularities of $S / \mathbb{D}_{8}$ are $4 A_{1}+A_{8,3}+A_{8,5}$. The resolution $Z$ of the quotient surface has invariants

$$
K_{Z}^{2}=-1, \quad c_{2}(Z)=13, \quad q=p_{g}=0 .
$$

Proof. Let $t$ and $a$ be generators of $\mathbb{D}_{8}$ such that $t^{8}=a^{2}=1$ and $a t=t^{7} a$. Order 8 elements in $G$ are $t, t^{3}, t^{5}, t^{7}$; order 4 elements are $t^{2}, t^{6}$; order 2 elements are $a$, ta, $t^{2} a, t^{3} a, t^{4} a, t^{5} a, t^{6} a, t^{7} a$ and $t^{4}$.

By the discussion on order 8 elements, $t$ has 4 fixed points, say $p_{1}, a p_{1}, p_{2}, a p_{2}$. Let $p$ be a fixed point of an involution $\sigma \neq t^{4}$. The orbit of $p$ under $G$ consists of 8 elements, each is a fixed point of an involution $\neq t^{4}$. The quotient surface has $\frac{1}{8} \cdot 8 \cdot 4 A_{1}+A_{8,3}+A_{8,5}$ singularities. We have

$$
e(S / G)=\frac{1}{16}(4+1 \cdot(8 \cdot 4)+7 \cdot 4)=4,
$$

and $e(Z)=4+4+2+3=13$. Moreover $K_{Z}^{2}=\frac{8}{16}-1-\frac{1}{2}=-1$.

\section{Reconstruction of a surface knowing its quotient}

Miyaoka [1984] gives a bound on the number of disjoint (-2)-curves on a minimal smooth surface $Y$. This implies in particular that if $c_{1}^{2}=4,2$ or 1 and $\chi\left(\mathcal{O}_{Y}\right)=1$, there are at most 4, 6 and 7 such curves respectively. The surfaces with $c_{1}^{2}=4,2$ we obtained as quotient of quaternionic fake quadrics reach that bound. For the cases $c_{1}^{2}=2$ these surfaces seem to be the first known ones with that property.

Dolgachev, Mendes Lopes, and Pardini [2002] study rational surfaces with the maximal number of $(-2)$-curves. For that aim they use and develop the theory of $(\mathbb{Z} / 2 \mathbb{Z})^{n}$-covers ramified over $A_{1}$ singularities. Using their results, we obtain:

Proposition 6.36. Let $Y$ be a smooth minimal surface of general type with $q=$ $p_{g}=0$ and ${ }_{2} \operatorname{Pic}(Y)=0$.

(a) If $c_{1}(Y)^{2}=4, c_{2}(Y)=8$ and $Y$ contains 4 disjoint (-2)-curves $C_{1}, \ldots, C_{4}$, then there exists a double cover of $Y$ ramified over the curves $C_{i}$. The minimal model of this covering has invariants $c_{1}^{2}=2 c_{2}=8$ and $q \leq 1$.

(b) If $c_{1}(Y)^{2}=2, c_{2}(Y)=10$ and $Y$ contains 6 disjoint (-2)-curves $C_{1}, \ldots, C_{6}$, then there exists a bidouble cover of $Y$ ramified over the curves $C_{i}$. The minimal model of this covering has invariants $c_{1}^{2}=2 c_{2}=8$ and $q \leq 2$.

(c) If $c_{1}(Y)^{2}=1, c_{2}(Y)=11$ and $Y$ contains 7 disjoint (-2)-curves $C_{1}, \ldots, C_{7}$, then there exists a $(\mathbb{Z} / 2 \mathbb{Z})^{3}$-cover of $Y$ ramified over the curves $C_{i}$. The minimal model of this covering has invariants $c_{1}^{2}=2 c_{2}=8$ and $q \leq 2$. 
Let $\mathbb{F}_{2}$ be the field with 2 elements. Let $C_{1}, \ldots, C_{k}$ be $k(-2)$-curves on a smooth surface $Y$. Let

$$
\psi: \mathbb{F}_{2}^{k} \rightarrow \operatorname{Pic}(Y) \otimes \mathbb{F}_{2}
$$

be the homomorphism sending $v=\left(v_{1}, \ldots, v_{k}\right)$ to $\sum v_{i} C_{i}$. We say that the curve $C_{j}$ appears in the kernel $\operatorname{ker} \psi$ if there is a vector $v=\left(v_{1}, \ldots, v_{k}\right)$ in $\operatorname{ker} \psi$ such that $v_{j}=1$. For $v$ in $\operatorname{ker} \psi$, we denote by $L_{v}$ an element of $\operatorname{Pic}(Y)$ such that $2 L_{v}=\sum v_{i} C_{i}$ (we sometimes identify elements of $\mathbb{F}_{2}$ with 0,1 in $\mathbb{Z}$ ). We have:

Proposition 6.37 [Dolgachev et al. 2002, Proposition 2.3]. Suppose that ${ }_{2} \operatorname{Pic}(Y)$ is zero. There exists a unique smooth connected Galois cover $\pi: Z \rightarrow Y$ such that the Galois group of $\pi$ is $G=\operatorname{Hom}\left(\operatorname{ker} \psi, \mathbb{G}_{m}\right)$, the branch locus of $\pi$ is the union of the $C_{i}$ appearing in ker $\psi$ and the surface $\bar{Z}$ obtained by contracting the $(-1)$-curves over the (-2)-curves in $Y$ has invariants

$$
K_{\bar{Z}}^{2}=2^{r} K_{Y}^{2} c_{2}(\bar{Z})=\chi\left(\mathcal{O}_{\bar{Z}}\right)=2^{r} \chi\left(\mathcal{O}_{Y}\right)-k 2^{r-3} \quad \text { and } \quad \kappa(\bar{Z})=\kappa(Y),
$$

where $r=\operatorname{dim} V$.

Proof of Proposition 6.36. We have to prove that for our surface $Y$, $\operatorname{ker} \psi$ has the required dimension and that all the curves appear in $\operatorname{ker} \psi$. For $c_{1}^{2}(Y)=4$ and 2, we have $b_{2}(Y)=h^{1,1}(Y)=6,8$ and 9 respectively. As we supposed that ${ }_{2} \operatorname{Pic}(Y)=0$, the space $\operatorname{Pic}(Y) \otimes \mathbb{F}_{2}$ is $h^{1,1}$-dimensional. As $p_{g}=0$, it has moreover a nondegenerate intersection pairing and therefore the dimension of a totally isotropic space in $\operatorname{Pic}(Y) \otimes \mathbb{F}_{2}$ is at most $\left[h^{1,1} / 2\right]=3,4$, and 4 dimensional respectively. The image of $\psi$ is the totally isotropic space generated by the curves $C_{i}$, therefore the dimension $r$ of ker $\psi$ is at least 1, 2 and 3 respectively.

A smooth double cover of a surface with $n$ nodes can exist only if $n$ is divisible by 4 (see [Dolgachev et al. 2002]). Therefore the vectors $v=\left(v_{1}, \ldots, v_{k}\right)$ in ker $\psi$ (of dimension $\leq 7$ ) have weight 4 , that is, the number of indices $j$ such that $v_{j}=1$ is 4 .

In case (a), ker $\psi$ is one-dimensional, generated by $w_{1}=(1,1,1,1)$. For (b), as every vector in ker $\psi$ has weight 4 , by [Beauville 1980, Lemme 1], we have $k \geq 2^{r}-1$ and thus $r \leq 2$ and $r \leq 3$ respectively. Moreover, it is easy to check that in the case (b), the space ker $\psi$ is (up to permutation of the basis vectors) generated by $w_{1}=(1,1,1,1,0,0)$ and $w_{2}=(1,1,0,0,1,1)$.

In case (c) [Beauville 1980, Lemme 1] implies that ker $\psi$ is (up to permutation) generated by $w_{1}=(1,0,0,1,1,0,1), w_{2}=(0,1,0,1,0,1,1)$ and $w_{3}=$ $(0,0,1,0,1,1,1)$.

The surface $\bar{Z}$ obtained by contracting the (-1)-curves over the (-2)-curves $C_{i}$ is minimal because no surface with $c_{1}^{2}=3 c_{2}=9$ has an order 2 automorphism.

Let us give a bound on the irregularity. 
Lemma 6.38. Let $Y$ be a surface of general type with $\chi=1$ and $q=0$ containing a 2-divisible set of $4(-2)$-curves. Let $Y^{\prime} \rightarrow Y$ be the double cover. Then $q\left(Y^{\prime}\right) \leq 1$. Proof. As $q(Y)=0$, the involution $\sigma$ on $Y^{\prime}$ given by the cover $Y^{\prime} \rightarrow Y$ acts as multiplication by -1 on $H^{0}\left(Y^{\prime}, \Omega_{Y^{\prime}}\right)$. Therefore, $\sigma$ acts trivially on $\wedge^{2} H^{0}\left(Y^{\prime}, \Omega_{Y^{\prime}}\right)$. As $p_{g}(Y)=0$, the map $\wedge^{2} H^{0}\left(Y^{\prime}, \Omega_{Y^{\prime}}\right) \rightarrow H^{0}\left(Y^{\prime}, \wedge^{2} \Omega_{Y^{\prime}}\right)$ must be 0 . Let $Y^{\prime} \rightarrow Y^{\prime \prime}$ be the blow-down map of the $4(-1)$-curves over the 4 nodal curves of $Y$. If $q\left(Y^{\prime \prime}\right) \geq 1$, Castelnuovo-De Franchis Theorem implies that there is a fibration onto a curve $B$ of genus $q\left(Y^{\prime \prime}\right)$. By [Zucconi 2003], we get that $q\left(Y^{\prime \prime}\right) \leq 2$ and if $q\left(Y^{\prime \prime}\right)=2$, then $Y^{\prime \prime}$ is an étale bundle of genus 2 fibers onto a genus 2 curve $B$ and $K_{Y^{\prime \prime}}^{2}=8$. In that case, there is a commutative diagram

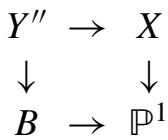

where the vertical maps are genus 2 fibrations and $X$ is the surface obtained by contracting the $4(-2)$-curves on $Y$. This diagram is obtained from $B \rightarrow \mathbb{P}^{1}$ by taking base change and normalizing. Since $Y^{\prime \prime} \rightarrow X$ is unramified in codimension 1, the 6 fibers of $X \rightarrow \mathbb{P}^{1}$ occurring at the 6 branch points of $B \rightarrow \mathbb{P}^{1}$ are double. Since $X$ has only 4 singular points, $X \rightarrow \mathbb{P}^{1}$ has at least two double fibers contained in the smooth locus of $X$, but a multiple fiber on a genus 2 fibration cannot exist (because of the adjunction formula). Thus $q \leq 1$.

Let us now consider a smooth minimal surface of general type $Z$ with $K^{2}=2$, $c_{2}=10, q=p_{g}=0$ such that there is a birational map onto a surface $Y$ with singularities $2 A_{3,1}+2 A_{3,2}$.

Proposition 6.39. Suppose that ${ }_{3} \operatorname{Pic}(Z)=0$. There exists a smooth triple cover $X$ of $Y$ ramified precisely over the singularities of $Y$. The surface $X$ is of general type and has invariants $c_{1}^{2}=2 c_{2}=8$.

Proof. Let $D_{1}, D_{2}$ be the (-3)-curves over the singularities $A_{3,1}$ and let $D_{3}, \ldots, D_{6}$ be the (-2)-curves over the singularities $A_{3,2}$, with indices satisfying $D_{3} D_{4}=$ $D_{5} D_{6}=1$. Let $W \rightarrow Y$ be the blow-up at the intersection points of $D_{3}, D_{4}$ and of $D_{5}, D_{6}$. Let $C_{1}, \ldots, C_{6}$ be the strict transforms of the $D_{i}$ in $W$. Let

$$
\psi: \mathbb{F}_{3}{ }^{6} \rightarrow \operatorname{Pic}(W) \otimes \mathbb{F}_{3}=H^{2}\left(W, \mathbb{F}_{3}\right)
$$

be the homomorphism sending $v=\left(v_{1}, \ldots, v_{k}\right)$ to $\sum v_{i} C_{i}$. The image of $\psi$ is a totally isotropic subspace in $H^{2}\left(W, \mathbb{F}_{3}\right)$. As $b_{2}(W)=10$, this image is at most 5-dimensional and therefore $\operatorname{dim} \operatorname{ker} \psi \geq 1$. Let $v=\left(v_{1}, \ldots, v_{6}\right) \in \operatorname{ker} \psi, v \neq 0$. We choose the representatives of $\mathbb{F}_{3}$ in $\{0,1,2\}$. There exists a unique invertible sheaf $L$ such that

$$
3 L=\sum v_{i} C_{i}
$$


Let $T$ be the triple cover of $W$ ramified over the $r$ curves $C_{i}$ such that $v_{i} \neq 0$. The surface $T$ is smooth outside the curves $C_{i}$ with $v_{i}=2$. Let $R$ be the minimal resolution of $T$ and let $f: R \rightarrow W$ be the composite map. By [Urzúa 2010, Propositions 2.2, 4.1 and 4.3], the invariants of $R$ are

$$
K_{R}={ }_{\text {num }} f^{*}\left(K_{W}+\frac{2}{3} \Sigma\right), \quad c_{2}(R)=3 c_{2}(W)-4 r, \quad \chi\left(\mathcal{O}_{R}\right)=3 \chi\left(\mathcal{O}_{W}\right)-\frac{1}{3} r,
$$

where $\Sigma$ is the sum of the $r$ curves $C_{i}$ such that $v_{i} \neq 0$. Therefore $r=3$ or 6 and

$$
K_{R}^{2}=0, \quad c_{2}(R)=36-4 r, \quad \chi\left(\mathcal{O}_{W}\right)=3-\frac{1}{3} r .
$$

As there are at least 3 curves $C_{i}$ in the branch locus, one of the curves $C_{3}, \ldots, C_{6}$ is in that branch locus. Say it is $C_{3}$. Let $E$ be the exceptional curve going through $C_{3}$. As $C_{3} E=C_{4} E=1$ and $E \sum v_{i} C_{i}$ is divisible by 3, it forces $C_{4}$ to be also in the branch locus and thus $r=6$ (and $\operatorname{dim} \operatorname{ker} \psi=1$ ). The inverse image of the 6 $(-3)$-curves are $(-1)$-curves. By the formula giving $K_{R}$, the inverse image of the two exceptional curves are $(-3)$-curves meeting two $(-1)$-curves. We can therefore effectuate 8 blow-downs and we obtain a fake quadric. It has general type because $Y$ has general type, it is minimal because the quotient of a fake plane by an order 3 automorphism with 4 isolated fixed points has $4 A_{2}$ singularities.

\section{Acknowledgements}

The authors warmly thank Fabrizio Catanese and Miles Reid for pointing out an error in a previous version. We are indebted to Fabrizio Catanese for his explanations on that point (see Remark 5.33 (a)) and to Håkan Granath for his help to correct that error. We thank also Ingrid Bauer, Margarida Mendes Lopes, and Rita Pardini for many useful discussions, and last but not least, we thank the referee for many useful comments and suggestions, in particular for the insistence to develop the $\mathbb{Q}$-homology quadrics point of view (see Remark 3.13). Part of this research was done during Roulleau's stay in Strasbourg University, and in the Instituto Superior Technico under grant FCT SFRH/BPD/72719/2010 and project Geometria Algebrica PTDC/MAT/099275/2008.

\section{References}

[Atiyah and Singer 1968] M. F. Atiyah and I. M. Singer, "The index of elliptic operators, III", Ann. of Math. (2) 87 (1968), 546-604. MR 38 \#5245 Zbl 0164.24301

[Barth et al. 2004] W. P. Barth, K. Hulek, C. A. M. Peters, and A. Van de Ven, Compact complex surfaces, 2nd ed., Ergebnisse der Mathematik und ihrer Grenzgebiete (3) 4, Springer, Berlin, 2004. MR 2004m:14070 Zbl 1036.14016

[Bauer et al. 2008] I. C. Bauer, F. Catanese, and F. Grunewald, "The classification of surfaces with $p_{g}=q=0$ isogenous to a product of curves", Pure Appl. Math. Q. 4:2, Part 1 (2008), 547-586. MR 2009a:14046 Zbl 1151.14027 
[Beauville 1980] A. Beauville, "Sur le nombre maximum de points doubles d'une surface dans $\mathbf{P}^{3}$ $(\mu(5)=31)$ ", pp. 207-215 in Journées de Géometrie Algébrique (Angers, 1979), edited by A. Beauville, Sijthoff \& Noordhoff, Alphen aan den Rijn, 1980. MR 82k:14037 Zbl 0445.14016

[Borel 1981] A. Borel, "Commensurability classes and volumes of hyperbolic 3-manifolds", Ann. Scuola Norm. Sup. Pisa Cl. Sci. (4) 8:1 (1981), 1-33. MR 82j:22008 Zbl 0473.57003

[Deuring 1968] M. Deuring, Algebren, 2nd ed., Ergebnisse der Mathematik und ihrer Grenzgebiete 41, Springer, Berlin, 1968. MR 37 \#4106 Zbl 0159.04201

[Dolgachev et al. 2002] I. Dolgachev, M. Mendes Lopes, and R. Pardini, "Rational surfaces with many nodes", Compositio Math. 132:3 (2002), 349-363. MR 2003g:14049 Zbl 1059.14050

[Džambić 2013] A. Džambić, "Fake quadrics from irreducible lattices acting on the product of upper half planes", preprint, 2013. arXiv 1305.5174

[Granath 2002] H. Granath, On quaternionic Shimura surfaces, thesis, Chalmers Tekniska Hogskola, Göteborg, 2002. MR 2715512

[Hirzebruch 1987] F. Hirzebruch, "Kommentare", pp. 755-804 in Gesammelte Abhandlungen, vol. I, Springer, Berlin, 1987. MR 89h:01106 Zbl 0627.01044

[Inose and Mizukami 1979] H. Inose and M. Mizukami, "Rational equivalence of 0-cycles on some surfaces of general type with $p_{g}=0$ ", Math. Ann. 244:3 (1979), 205-217. MR 82c:14009 Zbl 0444.14006

[Iskovskikh and Shafarevich 1989] V. A. Iskovskikh and I. R. Shafarevich, “Алгебраические поверхности", pp. 131-263 in Алгебраическая геометрия, II, edited by I. R. Shafarevich, Itogi Nauki Tekh., Ser. Sovrem. Probl. Mat., Fundam. Napravleniya 35, Akad. Nauk SSSR Vsesoyuz. Inst. Nauchn. i Tekhn. Inform., Moscow, 1989. Translated as "Algebraic surfaces" in Algebraic geometry, II, Encyclopaedia of Math. Sci. 35, 127-262, Springer, Berlin, 1996. MR 91f:14029 Zbl 0733.14015

[Keum 2006] J. Keum, "A fake projective plane with an order 7 automorphism”, Topology 45:5 (2006), 919-927. MR 2008b:14065 Zbl 1099.14031

[Keum 2008] J. Keum, "Quotients of fake projective planes”, Geom. Topol. 12:4 (2008), 2497-2515. MR 2009g:14042 Zbl 1222.14088

[Keum 2012] J. Keum, "Toward a geometric construction of fake projective planes", Atti Accad. Naz. Lincei Cl. Sci. Fis. Mat. Natur. Rend. Lincei (9) Mat. Appl. 23:2 (2012), 137-155. MR 2924897 Zbl 1248.14044

[Margulis 1991] G. A. Margulis, Discrete subgroups of semisimple Lie groups, Ergebnisse der Mathematik und ihrer Grenzgebiete (3) 17, Springer, Berlin, 1991. MR 92h:22021 Zbl 0732.22008

[Matsushima and Shimura 1963] Y. Matsushima and G. Shimura, "On the cohomology groups attached to certain vector valued differential forms on the product of the upper half planes", Ann. of Math. (2) 78 (1963), 417-449. MR 27 \#5274 Zbl 0141.38704

[Miyaoka 1984] Y. Miyaoka, "The maximal number of quotient singularities on surfaces with given numerical invariants", Math. Ann. 268:2 (1984), 159-171. MR 85j:14060 Zbl 0521.14013

[Prasad and Yeung 2012] G. Prasad and S.-K. Yeung, "Nonexistence of arithmetic fake compact Hermitian symmetric spaces of type other than $A_{n}(n \leq 4)$ ", J. Math. Soc. Japan 64:3 (2012), 683-731. MR 2965425 Zbl 1266.22015

[Riehm 1970] C. Riehm, "The norm 1 group of a p-adic division algebra", Amer. J. Math. 92 (1970), 499-523. MR 41 \#6860 Zbl 0199.37601

[Roulleau 2012] X. Roulleau, "Quotients of Fano surfaces", Atti Accad. Naz. Lincei Cl. Sci. Fis. Mat. Natur. Rend. Lincei (9) Mat. Appl. 23:3 (2012), 325-349. MR 2960841 Zbl 1252.14023 
[Shavel 1978] I. H. Shavel, "A class of algebraic surfaces of general type constructed from quaternion algebras”, Pacific J. Math. 76:1 (1978), 221-245. MR 58 \#28002 Zbl 0422.14022

[Shimizu 1963] H. Shimizu, "On discontinuous groups operating on the product of the upper half planes”, Ann. of Math. (2) 77 (1963), 33-71. MR 26 \#2641 Zbl 0218.10045

[Shimura 1967] G. Shimura, "Construction of class fields and zeta functions of algebraic curves", Ann. of Math. (2) 85 (1967), 58-159. MR 34 \#4268 Zbl 0204.07201

[Urzúa 2010] G. Urzúa, "Arrangements of curves and algebraic surfaces", J. Algebraic Geom. 19:2 (2010), 335-365. MR 2011e:14072 Zbl 1192.14033

[Vignéras 1980] M.-F. Vignéras, Arithmétique des algèbres de quaternions, Lecture Notes in Mathematics 800, Springer, Berlin, 1980. MR 82i:12016 Zbl 0422.12008

[Zhang 2001] D.-Q. Zhang, “Automorphisms of finite order on rational surfaces”, J. Algebra 238:2 (2001), 560-589. MR 2002c:14064 Zbl 1057.14053

[Zimmer 1984] R. J. Zimmer, Ergodic theory and semisimple groups, Monographs in Mathematics 81, Birkhäuser, Basel, 1984. MR 86j:22014 Zbl 0571.58015

[Zucconi 2003] F. Zucconi, "Surfaces with $p_{g}=q=2$ and an irrational pencil", Canad. J. Math. 55:3 (2003), 649-672. MR 2004d:14055 Zbl 1053.14042

Received January 18, 2012. Revised May 24, 2013.

AMIR DŽAMBIĆ

INSTITUT FÜR MATHEMATIK

JOHANN WOLFGANG GOETHE UNIVERSITÄT

ROBERT-MAYER-STR. 6-8

D-60325 FRANKFURT AM MAIN

GERMANY

dzambic@math.uni-frankfurt.de

XAVIER Roulleau

LABORATOIRE DE MATHÉMATIQUES ET APPliCATIONS

UNIVERSITÉ DE POITIERS

TÉLÉPORT 2 - BP 30179

86962 Futuroscope CHASSENEUIL

FRANCE

roulleau@math.univ-poitiers.fr 


\title{
PACIFIC JOURNAL OF MATHEMATICS
}

\author{
msp.org/pjm
}

Founded in 1951 by E. F. Beckenbach (1906-1982) and F. Wolf (1904-1989)

\section{EDITORS}

V. S. Varadarajan (Managing Editor)

Department of Mathematics

University of California

Los Angeles, CA 90095-1555

pacific@math.ucla.edu

Paul Balmer

Department of Mathematics

University of California

Los Angeles, CA 90095-1555

balmer@math.ucla.edu

Daryl Cooper

Department of Mathematics

University of California

Santa Barbara, CA 93106-3080 cooper@math.ucsb.edu

Jiang-Hua $\mathrm{Lu}$

Department of Mathematics

Pokfulam Rd., Hong Kong jhlu@maths.hku.hk
The University of Hong Kong

Don Blasius

Department of Mathematics University of California

Los Angeles, CA 90095-1555

blasius@math.ucla.edu

Robert Finn

Department of Mathematics Stanford University

Stanford, CA 94305-2125

finn@math.stanford.edu

Sorin Popa

Department of Mathematics

University of California

Los Angeles, CA 90095-1555 popa@math.ucla.edu

Paul Yang

Department of Mathematics Princeton University

Princeton NJ 08544-1000

yang@math.princeton.edu

\section{PRODUCTION}

Silvio Levy, Scientific Editor, production@msp.org

\section{SUPPORTING INSTITUTIONS}

ACADEMIA SINICA, TAIPEI

CALIFORNIA INST. OF TECHNOLOGY

INST. DE MATEMÁTICA PURA E APLICADA

KEIO UNIVERSITY

MATH. SCIENCES RESEARCH INSTITUTE

NEW MEXICO STATE UNIV.

OREGON STATE UNIV.

\author{
STANFORD UNIVERSITY \\ UNIV. OF BRITISH COLUMBIA \\ UNIV. OF CALIFORNIA, BERKELEY \\ UNIV. OF CALIFORNIA, DAVIS \\ UNIV. OF CALIFORNIA, LOS ANGELES \\ UNIV. OF CALIFORNIA, RIVERSIDE \\ UNIV. OF CALIFORNIA, SAN DIEGO \\ UNIV. OF CALIF., SANTA BARBARA
}

\author{
Vyjayanthi Chari \\ Department of Mathematics \\ University of California \\ Riverside, CA 92521-0135 \\ chari@math.ucr.edu \\ Kefeng Liu \\ Department of Mathematics \\ University of California \\ Los Angeles, CA 90095-1555 \\ liu@math.ucla.edu \\ Jie Qing \\ Department of Mathematics \\ University of California \\ Santa Cruz, CA 95064 \\ qing@cats.ucsc.edu
}

These supporting institutions contribute to the cost of publication of this Journal, but they are not owners or publishers and have no responsibility for its contents or policies.

See inside back cover or msp.org/pjm for submission instructions.

The subscription price for 2014 is US \$410/year for the electronic version, and \$535/year for print and electronic.

Subscriptions, requests for back issues and changes of subscribers address should be sent to Pacific Journal of Mathematics, P.O. Box 4163, Berkeley, CA 94704-0163, U.S.A. The Pacific Journal of Mathematics is indexed by Mathematical Reviews, Zentralblatt MATH, PASCAL CNRS Index, Referativnyi Zhurnal, Current Mathematical Publications and Web of Knowledge (Science Citation Index).

The Pacific Journal of Mathematics (ISSN 0030-8730) at the University of California, c/o Department of Mathematics, 798 Evans Hall \#3840, Berkeley, CA 94720-3840, is published twelve times a year. Periodical rate postage paid at Berkeley, CA 94704, and additional mailing offices. POSTMASTER: send address changes to Pacific Journal of Mathematics, P.O. Box 4163, Berkeley, CA 94704-0163.

PJM peer review and production are managed by EditFLOW ${ }^{\circledR}$ from Mathematical Sciences Publishers.

\section{PUBLISHED BY}

mathematical sciences publishers

nonprofit scientific publishing

http://msp.org/

(C) 2014 Mathematical Sciences Publishers 


\section{PACIFIC JOURNAL OF MATHEMATICS}

Volume $267 \quad$ No. $1 \quad$ January 2014

Numerical study of unbounded capillary surfaces

YASUNORI AOKI and HANS DE STERCK

Dual $R$-groups of the inner forms of $\operatorname{SL}(N)$

KUOK FAI CHAO and WEN-WEI LI

Automorphisms and quotients of quaternionic fake quadrics

AMIR DŽAmBIĆ and XAVIER ROULLEAU

Distance of bridge surfaces for links with essential meridional spheres 121

YEONHEE JANG

Normal states of type III factors

YASUYUKI KAWAHIGASHI, YOSHIKO OGATA and ERLING

STØRMER

Eigenvalues and entropies under the harmonic-Ricci flow

YI LI

Quantum extremal loop weight modules and monomial crystals

MATHIEU MANSUY

Lefschetz fibrations with small slope

NAOYUKI MONDEN 